TRANSACTIONS OF THE

AMERICAN MATHEMATICAL SOCIETY

Volume 365, Number 6, June 2013, Pages 3255-3286

S 0002-9947(2012)05738-2

Article electronically published on August 21, 2012

\title{
SOME THEOREMS ON FELLER PROCESSES: TRANSIENCE, LOCAL TIMES AND ULTRACONTRACTIVITY
}

\author{
RENÉ L. SCHILLING AND JIAN WANG
}

\begin{abstract}
We present sufficient conditions for the transience and the existence of local times of a Feller process, and the ultracontractivity of the associated Feller semigroup; these conditions are sharp for Lévy processes. The proof uses a local symmetrization technique and a uniform upper bound for the characteristic function of a Feller process. As a by-product, we obtain for stable-like processes (in the sense of $\mathrm{R}$. Bass) on $\mathbb{R}^{d}$ with smooth variable index $\alpha(x) \in(0,2)$ a transience criterion in terms of the exponent $\alpha(x)$; if $d=1$ and $\inf _{x \in \mathbb{R}} \alpha(x) \in(1,2)$, then the stable-like process has local times.
\end{abstract}

\section{BACKGROUND AND MAIN RESUlts}

In this paper we study sample path properties of Feller processes. Our main tool will be the fact that the infinitesimal generator of the associated Feller semigroup can be written as a pseudodifferential operator with negative definite symbol.

A Feller process $\left(X_{t}\right)_{t \geqslant 0}$ with state space $\mathbb{R}^{d}$ is a strong Markov process whose associated operator semigroup $\left(T_{t}\right)_{t \geqslant 0}$,

$$
T_{t} u(x)=\mathbb{E}^{x}\left(u\left(X_{t}\right)\right), \quad u \in C_{\infty}\left(\mathbb{R}^{d}\right), t \geqslant 0, x \in \mathbb{R}^{d},
$$

$\left(C_{\infty}\left(\mathbb{R}^{d}\right)\right.$ is the space of continuous functions vanishing at infinity) enjoys the Feller property, i.e. maps $C_{\infty}\left(\mathbb{R}^{d}\right)$ into itself. The semigroup $\left(T_{t}\right)_{t \geqslant 0}$ is said to be a Feller semigroup. That is, $\left(T_{t}\right)_{t \geqslant 0}$ is a one-parameter semigroup of contraction operators $T_{t}: C_{\infty}\left(\mathbb{R}^{d}\right) \rightarrow C_{\infty}\left(\mathbb{R}^{d}\right)$ which is strongly continuous: $\lim _{t \rightarrow 0}\left\|T_{t} u-u\right\|_{\infty}=0$ and has the sub-Markov property: $0 \leqslant T_{t} u \leqslant 1$ whenever $0 \leqslant u \leqslant 1$.

The (infinitesimal) generator $(A, \mathcal{D}(A))$ of the semigroup or the process is given by the strong limit

$$
A u:=\lim _{t \rightarrow 0} \frac{T_{t} u-u}{t}
$$

on the set $\mathcal{D}(A) \subset C_{\infty}\left(\mathbb{R}^{d}\right)$ of those $u \in C_{\infty}\left(\mathbb{R}^{d}\right)$ for which the above limit exists w.r.t. the sup-norm. We will call $(A, \mathcal{D}(A))$ Feller generator for short.

Before we proceed with general Feller semigroups it is instructive to have a brief look at Lévy processes and convolution semigroups, which are a particular subclass of Feller processes. Our standard reference for Lévy processes is the monograph by Sato [27. A Lévy process $\left(Y_{t}\right)_{t \geqslant 0}$ is a stochastically continuous random process with stationary and independent increments. The characteristic function of a Lévy

Received by the editors August 16, 2011 and, in revised form, October 31, 2011.

2010 Mathematics Subject Classification. Primary 60J25, 60J75, 35S05.

Key words and phrases. Feller process, characteristic function, symbol, (local) symmetrization, stable-like process, ultracontractivity, transience, local time. 
process has a particularly simple structure,

$$
\mathbb{E}^{x}\left(e^{i\left\langle\xi, Y_{t}-x\right\rangle}\right)=\mathbb{E}^{0}\left(e^{i\left\langle\xi, Y_{t}\right\rangle}\right)=e^{-t \psi(\xi)},
$$

where $\psi: \mathbb{R}^{d} \rightarrow \mathbb{C}$ is the characteristic exponent, which is a continuous negative definite function in the sense of Schoenberg. This amounts to saying that $\psi$ is given by the Lévy-Khintchine formula

$$
\psi(\xi)=c-i\langle b, \xi\rangle+\langle\xi, a \xi\rangle+\int_{y \neq 0}\left(1-e^{i\langle y, \xi\rangle}+i\langle y, \xi\rangle \mathbb{1}_{\{|y| \leqslant 1\}}\right) \nu(d y) .
$$

The tuple $(c, b, a, \nu)$ consisting of $c \in \mathbb{R}_{+}, b \in \mathbb{R}^{d}$, a positive semidefinite matrix $a \in \mathbb{R}^{d \times d}$ and a Radon measure $\nu$ on $\mathbb{R}^{d} \backslash\{0\}$ with $\int_{y \neq 0}|y|^{2}\left(1+|y|^{2}\right)^{-1} \nu(d y)<\infty$ is called Lévy characteristics (of $\psi$ ). The measure $\nu$ is often called Lévy measure. The Lévy characteristics determine $\psi$ uniquely - and vice versa. Since $\left(Y_{t}\right)_{t \geqslant 0}$ is a Markov process, (1.1) and (1.2) characterize the finite-dimensional distributions of $\left(Y_{t}\right)_{t \geqslant 0}$ and, hence, the process itself.

In the analysis of the Lévy process, the characteristic exponent $\psi$ and the fact that $\mathbb{E} e^{i\left\langle\xi, X_{t}\right\rangle}=e^{-t \psi(\xi)}$ play a key role. They allow, in particular, the use of methods from harmonic analysis to study Lévy processes. For details we refer to Sato's monograph [27] and the still unsurpassed survey paper by Fristedt [12. At this point let us just recall those properties which are relevant for the present paper.

(A) Existence of transition densities. The problem to decide whether a Lévy process $\left(Y_{t}\right)_{t \geqslant 0}$ has a transition density with respect to Lebesgue measure is not yet completely solved. The classic Hartman-Wintner condition

$$
\lim _{|\xi| \rightarrow \infty} \frac{\operatorname{Re} \psi(\xi)}{\log (1+|\xi|)}=\infty
$$

is sufficient, see [14, and necessary, see [21], for the existence of smooth densities for all times $t>0$. On the other hand, Hawkes' result 15 states that a Lévy process has for all $t>0$ a transition density if, and only if, $\left(Y_{t}\right)_{t \geqslant 0}$ has the strong Feller property.

(B) Transience and recurrence. A Markov process $\left(X_{t}\right)_{t \geqslant 0}$ is transient if there exists a countable cover $\left\{A_{j}\right\}_{j \geqslant 1}$ of $\mathbb{R}^{d}$ such that $\mathbb{E}^{x}\left(\int_{0}^{\infty} \mathbb{1}_{A_{j}}\left(X_{t}\right) d t\right)<\infty$ for every $x \in \mathbb{R}^{d}$ and $j \geqslant 1$; otherwise it is called recurrent. For a Lévy process $\left(Y_{t}\right)_{t \geqslant 0}$ we have the classic Chung-Fuchs criterion; see [7, 26] or [27, Section 37]: $\left(Y_{t}\right)_{t \geqslant 0}$ is transient or recurrent according to

$$
\int_{|\xi| \leqslant r} \frac{d \xi}{\operatorname{Re} \psi(\xi)}<\infty \quad \text { or } \quad=\infty
$$

for some, hence all, $r>0$.

(C) Local times. Let $\left(X_{t}\right)_{t \geqslant 0}$ be a Markov process on $\mathbb{R}^{d}$ and $\mathcal{F}_{t}:=\sigma\left(X_{s}: s \leqslant t\right)$. If there exists an $\left(\mathcal{F}_{t}\right)_{t \geqslant 0}$-adapted nonnegative process $(L(\cdot, t))_{t \geqslant 0}$ such that for any measurable bounded function $f \geqslant 0$,

$$
\int_{0}^{t} f\left(X_{s}\right) d s=\int_{\mathbb{R}^{d}} f(x) L(x, t) d x \quad \text { almost surely, }
$$

then $(L(\cdot, t))_{t \geqslant 0}$ is called the local time of the process. 
A Lévy process admits local times if, and only if, Hawkes' criterion is satisfied:

$$
\int_{\mathbb{R}^{d}} \frac{d \xi}{1+\operatorname{Re} \psi(\xi)}<\infty
$$

see [16, Theorems 1 and 3] or the earlier related result [13, Theorem 4].

Every Lévy process is spatially homogeneous. Therefore, the associated semigroup is of convolution type,

$$
S_{t} u(x)=\mathbb{E}^{x}\left(u\left(Y_{t}\right)\right)=\mathbb{E}^{0}\left(u\left(Y_{t}+x\right)\right)=\int u(x+y) \mathbb{P}^{0}\left(Y_{t} \in d y\right)
$$

and a short direct calculation shows that $\left(S_{t}\right)_{t \geqslant 0}$ is indeed a Feller semigroup with infinitesimal generator

$$
-\psi(D) u(x):=-\int \psi(\xi) \widehat{u}(\xi) e^{i\langle x, \xi\rangle} d \xi, \quad u \in C_{c}^{\infty}\left(\mathbb{R}^{d}\right),
$$

where $C_{c}^{\infty}\left(\mathbb{R}^{d}\right)$ is the space of smooth, compactly supported functions in $\mathbb{R}^{d}$ and $\widehat{u}(\xi):=(2 \pi)^{-d} \int u(x) e^{-i\langle x, \xi\rangle} d x$ denotes the Fourier transform of $u$; cf. [20, p. 141]. The operator $\psi(D)$ is a first example of a so-called pseudodifferential operator with symbol $\psi(\xi)$. Since $\psi$ does not depend on $x$ the operator has constant "coefficients". Notice that the symbol is just the characteristic exponent of the process $\left(Y_{t}\right)_{t \geqslant 0}$.

Let us return to the general situation. Under the assumption that the test functions $C_{c}^{\infty}\left(\mathbb{R}^{d}\right)$ are contained in $D(A)$, Ph. Courrège [8, Theorem 3.4] proved that the generator $A$ restricted to $C_{c}^{\infty}\left(\mathbb{R}^{d}\right)$ is a pseudodifferential operator,

$$
A u(x)=-p(x, D) u(x):=-\int e^{i\langle x, \xi\rangle} p(x, \xi) \hat{u}(\xi) d \xi, \quad u \in C_{c}^{\infty}\left(\mathbb{R}^{d}\right),
$$

with symbol $p: \mathbb{R}^{d} \times \mathbb{R}^{d} \rightarrow \mathbb{C}$. The symbol $p(x, \xi)$ is locally bounded in $(x, \xi)$, measurable as a function of $x$, and for every fixed $x \in \mathbb{R}^{d}$, it is a continuous negative definite function in the co-variable. This is to say that it enjoys the following LévyKhintchine representation,

$$
p(x, \xi)=c(x)-i\langle b(x), \xi\rangle+\frac{1}{2}\langle\xi, a(x) \xi\rangle+\int_{z \neq 0}\left(1-e^{i\langle z, \xi\rangle}+i\langle z, \xi\rangle \mathbb{1}_{\{|z| \leqslant 1\}}\right) \nu(x, d z),
$$

where $(c(x), b(x), a(x), \nu(x, d z))_{x \in \mathbb{R}^{d}}$ are the Lévy characteristics: $c(x)$ is a nonnegative measurable function, $b(x):=\left(b_{j}(x)\right) \in \mathbb{R}^{d}$ is a measurable function, $a(x):=\left(a_{j k}(x)\right) \in \mathbb{R}^{d \times d}$ is a nonnegative definite matrix-valued function, and $\nu(x, d z)$ is a nonnegative, $\sigma$-finite kernel on $\mathbb{R}^{d} \times \mathcal{B}\left(\mathbb{R}^{d} \backslash\{0\}\right)$ such that for every $x \in \mathbb{R}^{d}, \int_{z \neq 0}\left(1 \wedge|z|^{2}\right) \nu(x, d z)<+\infty$. For details and a comprehensive bibliography we refer to the monographs [18] by N. Jacob and the survey paper [20].

This means that Feller generators are "variable coefficient" Lévy-type operators and that, as soon as we fix $x=x_{0},-p\left(x_{0}, D\right)$ is the generator of a Lévy process. The problem is that $p(x, \xi)$ is no longer the characteristic exponent of the Feller process $\left(X_{t}\right)_{t \geqslant 0}$; i.e., the formula $\mathbb{E}^{x} e^{i\left\langle\xi, X_{t}-x\right\rangle}=e^{-t p(x, \xi)}$ is, in general, wrong. On the other hand, it is natural to expect that

$$
\mathbb{E}^{x} e^{i\left\langle\xi, X_{t}-x\right\rangle} \approx e^{-t p(x, \xi)}
$$

this would be quite clear for classical pseudodifferential operators (but the classical theory does not apply here since our symbols are "rough" and do not fit into that framework); see e.g. Taylor [35] and also [19] and [18]. The first major result of 
the present paper is, therefore, to establish a comparison between the characteristic function of the process and the symbol.

We will frequently make the following two assumptions on the symbol $p(x, \xi)$ :

$$
\|p(\cdot, \xi)\|_{\infty} \leqslant c\left(1+|\xi|^{2}\right) \quad \text { and } \quad p(\cdot, 0) \equiv 0 .
$$

The first condition means that the generator has only bounded 'coefficients'; see, e.g., [31, Lemma 2.1] or [32, Lemma 6.2]; the second condition implies that the Feller process is conservative in the sense that the life time of the process is almost surely infinite; see [30, Theorem 5.2].

Theorem 1.1. Let $\left(X_{t}\right)_{t \geqslant 0}$ be a Feller process with the generator $(A, D(A))$ such that $C_{c}^{\infty}\left(\mathbb{R}^{d}\right) \subset D(A)$. Then $\left.A\right|_{C_{c}^{\infty}\left(\mathbb{R}^{d}\right)}=-p(\cdot, D)$ is a pseudodifferential operator with symbol $p(x, \xi)$. Assume that the symbol satisfies (1.6). Then for any $t \geqslant 0$ and every $x, \xi \in \mathbb{R}^{d}$,

$$
\left|\mathbb{E}^{x}\left(e^{i\left\langle X_{t}-x, \xi\right\rangle}\right)\right| \leqslant \exp \left[-\frac{t}{16} \inf _{z \in \mathbb{R}^{d}} \operatorname{Re} p(z, 2 \xi)\right] .
$$

Note that the estimate (1.7) from Theorem 1.1 is both natural and trivial for a Lévy process $\left(Y_{t}\right)_{t \geqslant 0}$ :

$$
\left|\mathbb{E}^{x}\left(e^{i\left\langle Y_{t}-x, \xi\right\rangle}\right)\right|=\left|\mathbb{E}^{0}\left(e^{i\left\langle Y_{t}, \xi\right\rangle}\right)\right|=\left|e^{-t \psi(\xi)}\right|=e^{-t \operatorname{Re} \psi(\xi)} .
$$

With the help of Theorem 1.1 we are able to provide criteria for the ultracontractivity of Feller semigroups, the transience and the existence of local times of Feller processes. The results (i)-(iii) from Theorem 1.2 below are the exact counterparts of the corresponding results and criteria $(\mathrm{A})-(\mathrm{C})$ for a Lévy process. In particular, this indicates that the results are sharp.

Theorem 1.2. Let $\left(X_{t}\right)_{t \geqslant 0}$ be a Feller process with the generator $(A, D(A))$ such that $C_{c}^{\infty}\left(\mathbb{R}^{d}\right) \subset D(A)$. Then $\left.A\right|_{C_{c}^{\infty}\left(\mathbb{R}^{d}\right)}=-p(\cdot, D)$ is a pseudodifferential operator with symbol $p(x, \xi)$. Assume that the symbol satisfies (1.6).

(i) If

$$
\lim _{|\xi| \rightarrow \infty} \frac{\inf _{z \in \mathbb{R}^{d}} \operatorname{Re} p(z, \xi)}{\log (1+|\xi|)}=\infty,
$$

then the corresponding Feller semigroup $\left(T_{t}\right)_{t \geqslant 0}$ is ultracontractive; i.e., $\left\|T_{t}\right\|_{1 \rightarrow \infty}<$ $\infty$ for every $t>0$.

If $P(t, x, d y)$ is the transition function of $\left(X_{t}\right)_{t \geqslant 0}$, then $P(t, x, d y)$ has a density function $p(t, x, y)$ with respect to Lebesgue measure, and for every $t>0$,

$$
\sup _{x, y \in \mathbb{R}^{d}} p(t, x, y) \leqslant(4 \pi)^{-d} \int \exp \left(-\frac{t}{16} \inf _{z \in \mathbb{R}^{d}} \operatorname{Re} p(z, \xi)\right) d \xi .
$$

Consequently, the Feller semigroup $\left(T_{t}\right)_{t \geqslant 0}$ has the strong Feller property; i.e., for any $f \in B_{b}\left(\mathbb{R}^{d}\right)$ and $t>0, T_{t} f \in C_{b}\left(\mathbb{R}^{d}\right)$, where $C_{b}\left(\mathbb{R}^{d}\right)$ is the space of bounded continuous functions on $\mathbb{R}^{d}$.

(ii) If

$$
\int_{\{|\xi| \leqslant r\}} \frac{d \xi}{\inf _{z \in \mathbb{R}^{d}} \operatorname{Re} p(z, \xi)}<\infty \text { for every } r>0,
$$

then the Feller process $\left(X_{t}\right)_{t \geqslant 0}$ is transient. 
(iii) If

$$
\int_{\mathbb{R}^{d}} \frac{d \xi}{1+\inf _{z \in \mathbb{R}^{d}} \operatorname{Re} p(z, \xi)}<\infty,
$$

then the Feller process $\left(X_{t}\right)_{t \geqslant 0}$ has local times $(L(\cdot, t))_{t \geqslant 0}$ on $L^{2}(d x \otimes d \mathbb{P})$.

The rest of this paper is organized as follows. In Section 2 we will study the characteristic function of a Feller process. We first point out that, under some mild additional assumptions on a Feller process, the characteristic function is real if, and only if, the associated symbol is real. Then, we give the proof of Theorem 1.1 by using the local symmetrization technique; this approach may well turn out to be useful for further studies of Feller processes. Section 3 is devoted to proving Theorem 1.2. Some examples, including stable-like processes, are presented here to illustrate our results. For the sake of completeness, a few necessary properties and estimates for a Feller process are proved in a simple and self-contained way in the appendix.

\section{Characteristic Functions of Feller processes}

Before we study the characteristic functions of Feller processes, it is instructive to have a brief look at Lévy processes which are a particular subclass of Feller processes. Our standard reference for Lévy processes is the monograph by K. Sato [27. A Lévy process $\left(Y_{t}\right)_{t \geqslant 0}$ is a stochastically continuous random process with stationary and independent increments. The characteristic function of a Lévy process has a particularly simple structure,

$$
\mathbb{E}^{x}\left(e^{i\left\langle Y_{t}-x, \xi\right\rangle}\right)=\mathbb{E}^{0}\left(e^{i\left\langle Y_{t}, \xi\right\rangle}\right)=e^{-t \psi(\xi)}, \quad x, \xi \in \mathbb{R}^{d}, t \geqslant 0,
$$

where $\psi: \mathbb{R}^{d} \rightarrow \mathbb{C}$ is a continuous negative definite function; i.e., it is given by a Lévy-Khintchine formula of the form (1.5) with characteristics $(c, b, a, \nu(d z))$ which do not depend on $x$. A short direct calculation shows that the infinitesimal generator of $Y_{t}$ is given by

$$
L u(x)=-\psi(D) u(x):=-\int e^{i\langle x, \xi\rangle} \psi(\xi) \hat{u}(\xi) d \xi, \quad u \in C_{c}^{\infty}\left(\mathbb{R}^{d}\right) .
$$

This means that a Lévy process is generated by a constant-coefficient pseudodifferential operator. The symbol is given by the characteristic exponent (i.e. the logarithm of the characteristic functions) of the Lévy process.

This relation is no longer true for general Feller processes. Since the Feller process $\left(X_{t}\right)_{t \geqslant 0}$ is not spatially homogeneous, the characteristic function of $X_{t}, t \geqslant 0$, will now depend on the starting point $x \in \mathbb{R}^{d}$, i.e. on $\mathbb{P}^{x}$. Therefore, we get a $(d+1)$ parameter family of characteristic functions:

$$
\lambda_{t}(x, \xi):=e^{-i\langle\xi, x\rangle} T_{t}\left(e^{i\langle\xi, \cdot\rangle}\right)(x)=\mathbb{E}^{x}\left(e^{i\left\langle X_{t}-x, \xi\right\rangle}\right) ;
$$

hence, for every $t \geqslant 0$ and $x \in \mathbb{R}^{d}$, the function $\xi \mapsto \lambda_{t}(x, \xi)$ is positive definite. Note that (2.12) is well defined, since the operator $T_{t}$ extends uniquely to a bounded operator on $B_{b}\left(\mathbb{R}^{d}\right)$ (the space of bounded measurable functions); cf. [30, Section 3]. According to [17, Theorem 1.1], for any Schwartz function $u$, we have

$$
T_{t} u(x)=\int e^{i\langle x, \xi\rangle} \hat{u}(\xi) \lambda_{t}(x, \xi) d \xi ;
$$


i.e. on the Schwartz space $\mathcal{S}\left(\mathbb{R}^{d}\right)$ the operator $T_{t}, t \geqslant 0$, is a pseudodifferential operator with symbol $\lambda_{t}(x, \xi)$.

If the domain of the Feller generator $A$ is sufficiently rich, e.g. if it contains the space $C_{b}^{2}\left(\mathbb{R}^{d}\right)$ of twice differentiable functions with bounded derivatives, we know from [17, Theorem 1.2] (and [29, Theorem 3.1] for the general case) that

$$
\left.\frac{d}{d t} \lambda_{t}(x, \xi)\right|_{t=0}=-p(x, \xi), \quad x, \xi \in \mathbb{R}^{d} .
$$

This allows us to interpret the symbol probabilistically as the derivative of the characteristic function of the process. Since the symbol of $T_{t}$ is not $e^{-t q(x, \xi)}$, we can only expect that the pseudodifferential operator $e^{-t q(x, D)}$ with symbol $e^{-t q(x, \xi)}$ is a reasonably good approximation. Under some mild additional assumptions on $p(x, \xi)$, one of us obtained in [28, Lemma 2] the following pointwise estimate:

$$
\left|\lambda_{t}(x, \xi)-e^{-t q(x, \xi)}\right| \leqslant C(\xi, \rho) t^{\rho}
$$

for $t \geqslant 0, \rho \in[0,1]$ and $x, \xi \in \mathbb{R}^{d}$. See also the earlier related paper [19].

2.1. Characteristic functions and symbols. Recall that $\left(\left(X_{t}\right)_{t \geqslant 0},\left(\mathbb{P}^{x}\right)_{x \in \mathbb{R}^{d}}\right)$ is a solution to the martingale problem for the operator $\left(-p(\cdot, D), C_{c}^{\infty}\left(\mathbb{R}^{d}\right)\right)$, if $\mathbb{P}^{x}\left(X_{0}=x\right)=1$ for all $x \in \mathbb{R}^{d}$, and if for all $f \in C_{c}^{\infty}\left(\mathbb{R}^{d}\right)$ the process $\left(M_{t}^{f}, \mathcal{F}_{t}\right)_{t \geqslant 0}$,

$$
M_{t}^{f}:=f\left(X_{t}\right)-\int_{0}^{t}\left(-p\left(X_{s}, D\right)\right) f\left(X_{s}\right) d s,
$$

is a local martingale under $\mathbb{P}^{x}$. Here $\mathcal{F}_{t}=\sigma\left(X_{s}: s \leqslant t\right)$ is the natural filtration of the process $\left(X_{t}\right)_{t \geqslant 0}$. The martingale problem for $\left(-p(\cdot, D), C_{c}^{\infty}\left(\mathbb{R}^{d}\right)\right)$ is well posed if the finite-dimensional distributions for any two solutions with the same initial distribution coincide.

The following result points out the relations between characteristic functions and the symbol of Feller processes.

Theorem 2.1. Let $\left(X_{t}\right)_{t \geqslant 0}$ be a Feller process with the generator $(A, D(A))$ such that $C_{c}^{\infty}\left(\mathbb{R}^{d}\right) \subset D(A)$. Then $\left.A\right|_{C_{c}^{\infty}\left(\mathbb{R}^{d}\right)}=-p(\cdot, D)$ is a pseudodifferential operator with symbol $p(x, \xi)$. For any $x \in \mathbb{R}^{d}$ and $t \geqslant 0$, let $\lambda_{t}(x, \xi)$ be the characteristic function of $\left(X_{t}\right)_{t \geqslant 0}$ given by (2.12). Assume that the symbol $p(x, \xi)$ satisfies (1.6). Then, we have the following statements:

(i) The assertion (2.14) holds; that is, for any $x, \xi \in \mathbb{R}^{d}$,

$$
\left.\frac{d}{d t} \lambda_{t}(x, \xi)\right|_{t=0}=-p(x, \xi) .
$$

(ii) If the characteristic function $\lambda_{t}(x, \xi)$ is real for all $x, \xi \in \mathbb{R}^{d}$ and $t \geqslant 0$, then the symbol $p(x, \xi)$ is also real.

(iii) Suppose that the martingale problem for $\left(-p(\cdot, D), C_{c}^{\infty}\left(\mathbb{R}^{d}\right)\right)$ is well posed. If the symbol $p(x, \xi)$ is real, then the characteristic function $\lambda_{t}(x, \xi)$ is real for all $x, \xi \in \mathbb{R}^{d}$ and $t \geqslant 0$.

Remark 2.2. The statement that the martingale problem for $\left(-p(\cdot, D), C_{c}^{\infty}\left(\mathbb{R}^{d}\right)\right)$ is well posed is equivalent to saying that the test functions $C_{c}^{\infty}\left(\mathbb{R}^{d}\right)$ are an operator core for the Feller operator $(A, D(A))$, i.e., $\overline{\left.A\right|_{C_{c}^{\infty}\left(\mathbb{R}^{d}\right)}}=A$. See Proposition 4.6 in the appendix for the proof.

We start with some analytic properties of a symbol $p(x, \xi)$ which satisfies (1.6). 
Lemma 2.3. Let $\left(X_{t}\right)_{t \geqslant 0}$ be a Feller process with the generator $(A, D(A))$ such that $C_{c}^{\infty}\left(\mathbb{R}^{d}\right) \subset D(A)$; i.e., $\left.A\right|_{C_{c}^{\infty}\left(\mathbb{R}^{d}\right)}=-p(\cdot, D)$ is a pseudodifferential operator with symbol $p(x, \xi)$. If the symbol $p(x, \xi)$ satisfies (1.6), then the function $x \mapsto p(x, \xi)$ is continuous for every fixed $\xi \in \mathbb{R}^{d}$, and

$$
\lim _{r \rightarrow 0} \sup _{z \in \mathbb{R}^{d}} \sup _{|\xi| \leqslant r}|p(z, \xi)|=0 .
$$

Proof. Since $C_{c}^{\infty}\left(\mathbb{R}^{d}\right) \subset D(A)$ and $\left.A\right|_{C_{c}^{\infty}\left(\mathbb{R}^{d}\right)}=-p(\cdot, D)$, the operator $-p(\cdot, D)$ maps $C_{c}^{\infty}\left(\mathbb{R}^{d}\right)$ into $C_{\infty}\left(\mathbb{R}^{d}\right)$. By the assumption (1.6), the function $x \mapsto p(x, 0)=0$ is continuous. Therefore, the required assertions follow from (the proof of) 30 , Theorem 4.4].

Proof of Theorem 2.1. (i) Under more restrictive conditions, the conclusion (2.14) has been shown in [17, Theorem 1.2] and [29, Theorem 3.1]. The following selfcontained proof avoids these technical restrictions.

Every Feller semigroup $\left(T_{t}\right)_{t \geqslant 0}$ has a unique extension onto the space $B_{b}\left(\mathbb{R}^{d}\right)$ of bounded Borel measurable functions; cf. [30, Section 3]. For notational simplicity, we use $\left(T_{t}\right)_{t \geqslant 0}$ for this extension. According to [30. Corollary 3.3 and Theorem 4.3] and Lemma 2.3, $t \mapsto T_{t} u$ is continuous with respect to locally uniform convergence for all continuous and bounded functions $u \in C_{b}\left(\mathbb{R}^{d}\right)$.

Let $e_{\xi}(x)=e^{i\langle\xi, x\rangle}$ for $x, \xi \in \mathbb{R}^{d}$. By Proposition 4.2 in the appendix, we know that for $t>0$ and $x, \xi \in \mathbb{R}^{d}$,

$$
T_{t} e_{\xi}(x)=e_{\xi}(x)+\int_{0}^{t} T_{s} A e_{\xi}(x) d s .
$$

Note that, see e.g. [31, Proof of Lemma 6.3, Page 607, Lines 14-15],

$$
-p(x, \xi)=e_{-\xi}(x) A e_{\xi}(x) .
$$

Therefore,

$$
\lambda_{t}(x, \xi)=e_{-\xi}(x) T_{t} e_{\xi}(x)=1-e^{-i\langle\xi, x\rangle} \int_{0}^{t} T_{s}\left(p(\cdot, \xi) e^{i\langle\xi, \cdot\rangle}\right)(x) d s .
$$

Since $\lambda_{0}(x, \xi)=1$, we obtain that for any $x, \xi \in \mathbb{R}^{d}$,

$$
\begin{aligned}
\left.\frac{d}{d t} \lambda_{t}(x, \xi)\right|_{t=0} & =\lim _{t \rightarrow 0} \frac{\lambda_{t}(x, \xi)-1}{t} \\
& =-e^{-i\langle\xi, x\rangle} \lim _{t \rightarrow 0} \frac{\int_{0}^{t} T_{s}\left(p(\cdot, \xi) e^{i\langle\xi, \cdot}\right)(x) d s}{t} \\
& =-e^{-i\langle\xi, x\rangle} p(x, \xi) e^{i\langle\xi, x\rangle} \\
& =-p(x, \xi) .
\end{aligned}
$$

In the third equality we have used the fact that for fixed $x, \xi \in \mathbb{R}^{d}$, the function $t \mapsto T_{t}\left(p(\cdot, \xi) e^{i\langle\xi, \cdot\rangle}\right)(x)$ is continuous; cf. the remark in the last paragraph. This proves (i).

(ii) This follows directly from (i).

(iii) For every $t \geqslant 0$ we define $\widetilde{X}_{t}=2 X_{0}-X_{t}$. Clearly, $\left(\widetilde{X}_{t}\right)_{t \geqslant 0}$ is also a strong Markov process with the same starting point as $\left(X_{t}\right)_{t \geqslant 0}$. Let $\widetilde{\mathbb{P}}^{x}$ be the probability of the process $\left(\widetilde{X}_{t}\right)_{t \geqslant 0}$ with starting point $x \in \mathbb{R}^{d}$, and denote by $\left(\widetilde{T}_{t}\right)_{t \geqslant 0}$ the semigroup of $\left(\widetilde{X}_{t}\right)_{t \geqslant 0}$. We claim that $\left(\widetilde{X}_{t}\right)_{t \geqslant 0}$ enjoys the Feller property. 
Let $P(t, x, d y)$ be the transition function of the process $\left(X_{t}\right)_{t \geqslant 0}$. Then, for all $u \in C_{\infty}\left(\mathbb{R}^{d}\right)$ and for a fixed $x_{0} \in \mathbb{R}^{d}$, we find

$$
\begin{aligned}
& \left|\widetilde{\mathbb{E}}^{x}\left(u\left(\widetilde{X}_{t}\right)\right)-\widetilde{\mathbb{E}}^{x_{0}}\left(u\left(\widetilde{X}_{t}\right)\right)\right| \\
& =\left|\mathbb{E}^{x}\left(u\left(2 x-X_{t}\right)\right)-\mathbb{E}^{x_{0}}\left(u\left(2 x_{0}-X_{t}\right)\right)\right| \\
& \leqslant\left|\mathbb{E}^{x}\left(u\left(2 x-X_{t}\right)\right)-\mathbb{E}^{x}\left(u\left(2 x_{0}-X_{t}\right)\right)\right|+\left|\mathbb{E}^{x}\left(u\left(2 x_{0}-X_{t}\right)\right)-\mathbb{E}^{x_{0}}\left(u\left(2 x_{0}-X_{t}\right)\right)\right| \\
& \leqslant \int\left|u(2 x-y)-u\left(2 x_{0}-y\right)\right| P(t, x, d y)+\left|\mathbb{E}^{x}\left(u\left(2 x_{0}-X_{t}\right)\right)-\mathbb{E}^{x_{0}}\left(u\left(2 x_{0}-X_{t}\right)\right)\right| .
\end{aligned}
$$

Since $u$ is uniformly continuous, we find for every $\epsilon>0$ some $\delta:=\delta(\varepsilon)>0$ such that $\left|u\left(z_{1}\right)-u\left(z_{2}\right)\right|<\epsilon$ for all $\left|z_{1}-z_{2}\right|<\delta$. This and the Feller property of $X_{t}$ show that for all $\left|x-x_{0}\right|<\delta$,

$$
\left|\widetilde{\mathbb{E}}^{x}\left(u\left(\widetilde{X}_{t}\right)\right)-\widetilde{\mathbb{E}}^{x_{0}}\left(u\left(\widetilde{X}_{t}\right)\right)\right| \leqslant \epsilon+\left|\mathbb{E}^{x}\left(u\left(2 x_{0}-X_{t}\right)\right)-\mathbb{E}^{x_{0}}\left(u\left(2 x_{0}-X_{t}\right)\right)\right| .
$$

We can now let $x \rightarrow x_{0}$ and obtain $\lim \sup _{x \rightarrow x_{0}}\left|\widetilde{\mathbb{E}}^{x}\left(u\left(\widetilde{X}_{t}\right)\right)-\widetilde{\mathbb{E}}^{x_{0}}\left(u\left(\widetilde{X}_{t}\right)\right)\right| \leqslant \epsilon$. Since $\epsilon>0$ was arbitrary, we can let $\epsilon \rightarrow 0$ and conclude that

$$
\lim _{x \rightarrow x_{0}}\left|\widetilde{\mathbb{E}}^{x}\left(u\left(\widetilde{X}_{t}\right)\right)-\widetilde{\mathbb{E}}^{x_{0}}\left(u\left(\widetilde{X}_{t}\right)\right)\right|=0 .
$$

On the other hand, let $\tau_{B(x, r)}$ be the first exit time of the process from the ball $B(x, r)$. According to Proposition 4.3 in the appendix, we know for all $r>0$ and $x \in \mathbb{R}^{d}$,

$$
\mathbb{P}^{x}\left(\left|X_{t}-x\right| \geqslant r\right) \leqslant \mathbb{P}^{x}\left(\tau_{B(x, r)} \leqslant t\right) \leqslant c_{1} t \sup _{z \in \mathbb{R}^{d}} \sup _{|\xi| \leqslant 1 / r}|p(z, \xi)|
$$

for some constant $c_{1}>0$. By the assertion (2.16) in Lemma 2.3, we can choose $\delta_{1}:=\delta_{1}(\varepsilon)$ such that

$$
\mathbb{P}^{x}\left(\left|X_{t}-x\right| \geqslant \delta_{1}\right) \leqslant \varepsilon /\left(2\|u\|_{\infty}\right) .
$$

Since $u \in C_{\infty}\left(\mathbb{R}^{d}\right)$, we find $\delta_{2}:=\delta_{2}(\varepsilon)>0$ such that $\sup _{|z| \geqslant \delta_{2}}|u(z)| \leqslant \varepsilon / 2$. Therefore, for all $t>0$ and $x \in \mathbb{R}^{d}$ with $|x| \geqslant \delta_{1}+\delta_{2}$, we have

$$
\begin{aligned}
\left|\widetilde{\mathbb{E}}^{x}\left(u\left(\widetilde{X}_{t}\right)\right)\right| & =\left|\mathbb{E}^{x}\left(u\left(2 x-X_{t}\right)\right)\right| \\
& \leqslant \mathbb{E}^{x}\left(\left|u\left(2 x-X_{t}\right)\right| \mathbb{1}_{\left\{\left|X_{t}-x\right| \leqslant \delta_{1}\right\}}\right)+\mathbb{E}^{x}\left(\left|u\left(2 x-X_{t}\right)\right| \mathbb{1}_{\left\{\left|X_{t}-x\right| \geqslant \delta_{1}\right\}}\right) \\
& \leqslant \sup _{|z| \geqslant|x|-\delta_{1}}|u(z)|+\|u\|_{\infty} \mathbb{P}^{x}\left(\left|X_{t}-x\right| \geqslant \delta_{1}\right) \\
& \leqslant \sup _{|z| \geqslant \delta_{2}}|u(z)|+\frac{\varepsilon}{2} \leqslant \varepsilon,
\end{aligned}
$$

which proves the Feller property of $\left(\widetilde{T}_{t}\right)_{t \geqslant 0}$.

Let $(\widetilde{A}, D(\widetilde{A}))$ be the generator of the Feller semigroup $\left(\widetilde{T}_{t}\right)_{t \geqslant 0}$. We claim that $C_{c}^{\infty}\left(\mathbb{R}^{d}\right) \subset D(\widetilde{A})$ and

$$
\left.\widetilde{A}\right|_{C_{c}^{\infty}\left(\mathbb{R}^{d}\right)}=-p(\cdot, D)=\left.A\right|_{C_{c}^{\infty}\left(\mathbb{R}^{d}\right)} .
$$

For this, we use the weak infinitesimal operator $\left(\widetilde{A}_{w}, D\left(\widetilde{A}_{w}\right)\right)$ of the Feller process $\left(\widetilde{X}_{t}\right)_{t \geqslant 0}$; see [9, Chapter I, Section 6] for details on the weak infinitesimal operator of a Markov semigroup. According to [27. Lemma 31.7, Page 209], we have $(\widetilde{A}, D(\widetilde{A}))=\left(\widetilde{A}_{w}, D\left(\widetilde{A}_{w}\right)\right)$. Therefore, it suffices to verify that the test functions 
$C_{c}^{\infty}\left(\mathbb{R}^{d}\right)$ are in the weak domain $D\left(\widetilde{A}_{w}\right)$ and that $\left.\widetilde{A}_{w}\right|_{C_{c}^{\infty}\left(\mathbb{R}^{d}\right)}=-p(\cdot, D)$. We have to show that for $u \in C_{c}^{\infty}\left(\mathbb{R}^{d}\right)$ and every $x \in \mathbb{R}^{d}$,

$$
\lim _{t \rightarrow 0} \frac{\widetilde{T}_{t} u(x)-u(x)}{t}=-p(x, D) u(x) .
$$

This can be seen from the following arguments. Using the Fourier transform, the Fubini theorem and the definition of $\left(\widetilde{X}_{t}\right)_{t \geqslant 0}$, we get

$$
\begin{aligned}
\lim _{t \rightarrow 0} & \frac{\widetilde{\mathbb{E}}^{x}\left(u\left(\widetilde{X}_{t}\right)-u(x)\right)}{t} \\
& =\lim _{t \rightarrow 0} \frac{1}{t} \widetilde{\mathbb{E}}^{x}\left(\int e^{i\left\langle\widetilde{X}_{t}, \xi\right\rangle} \hat{u}(\xi) d \xi-\int e^{i\langle x, \xi\rangle} \hat{u}(\xi) d \xi\right) \\
& =\lim _{t \rightarrow 0} \frac{1}{t} \int \hat{u}(\xi) \widetilde{\mathbb{E}}^{x}\left(e^{i\left\langle\widetilde{X}_{t}, \xi\right\rangle}-e^{i\langle x, \xi\rangle}\right) d \xi \\
& =\lim _{t \rightarrow 0} \frac{1}{t} \int \hat{u}(\xi) e^{i\langle 2 x, \xi\rangle} \mathbb{E}^{x}\left(e^{-i\left\langle X_{t}, \xi\right\rangle}-e^{-i\langle x, \xi\rangle}\right) d \xi \\
& =\lim _{t \rightarrow 0} \frac{1}{t} \int \hat{u}(\xi) e^{i\langle 2 x, \xi\rangle} \mathbb{E}^{x}\left(\int_{0}^{t} A e^{-i\left\langle X_{s}, \xi\right\rangle} d s\right) d \xi \\
& =\lim _{t \rightarrow 0} \frac{1}{t} \int \hat{u}(\xi) e^{i\langle 2 x, \xi\rangle} \mathbb{E}^{x}\left(\int_{0}^{t} e^{-i\left\langle X_{s}, \xi\right\rangle}\left(-p\left(X_{s},-\xi\right)\right) d s\right) d \xi \\
& =-\int e^{i\langle x, \xi\rangle} p(x,-\xi) \hat{u}(\xi) d \xi \\
& =-\int e^{i\langle x, \xi\rangle} p(x, \xi) \hat{u}(\xi) d \xi \\
& =-p(x, D) u(x) .
\end{aligned}
$$

In this calculation we have (repeatedly) used that $C_{c}^{\infty}\left(\mathbb{R}^{d}\right) \subset D(A),\left.A\right|_{C_{c}^{\infty}\left(\mathbb{R}^{d}\right)}=$ $-p(\cdot, D)$ and that the function $x \mapsto e_{\xi}(x)=e^{-i\langle x, \xi\rangle}$ belongs for every fixed $\xi \in \mathbb{R}^{d}$ to the extended domain of the Feller operator $\widetilde{D}(A)$; see Proposition 4.2 below. In the penultimate line we used that $p(x, \xi)$ is real, i.e. $p(x, \xi)=p(x,-\xi)$. Therefore, the weak infinitesimal operator of $\left(\widetilde{T}_{t}\right)_{t \geqslant 0}$ on $C_{c}^{\infty}\left(\mathbb{R}^{d}\right)$ is just $-p(\cdot, D)$.

According to [10, Chapter 4, Proposition 1.7] and [9, Chapter I, (1.49), Page 40], both $\left(\left(X_{t}\right)_{t \geqslant 0},\left(\mathbb{P}^{x}\right)_{x \in \mathbb{R}^{d}}\right)$ and $\left(\left(\widetilde{X}_{t}\right)_{t \geqslant 0},\left(\widetilde{\mathbb{P}}^{x}\right)_{x \in \mathbb{R}^{d}}\right)$ are solutions to the martingale problem for the operator $\left(-p(\cdot, D), C_{c}^{\infty}\left(\mathbb{R}^{d}\right)\right)$. Since the martingale problem for $\left(-p(\cdot, D), C_{c}^{\infty}\left(\mathbb{R}^{d}\right)\right)$ is well posed, $\left(\left(\widetilde{X}_{t}\right)_{t \geqslant 0},\left(\widetilde{\mathbb{P}}^{x}\right)_{x \in \mathbb{R}^{d}}\right)$ and $\left(\left(X_{t}\right)_{t \geqslant 0},\left(\mathbb{P}^{x}\right)_{x \in \mathbb{R}^{d}}\right)$ have the same finite-dimensional distributions. In particular, for any $t>0$ and $x, \xi \in \mathbb{R}^{d}$,

$$
\mathbb{E}^{x}\left(e^{i\left\langle X_{t}-x, \xi\right\rangle}\right)=\widetilde{\mathbb{E}}^{x}\left(e^{i\left\langle\widetilde{X_{t}}-x, \xi\right\rangle}\right),
$$

which shows that $\lambda_{t}(x, \xi)=\lambda_{t}(x,-\xi)=\overline{\lambda_{t}(x, \xi)}$; i.e., the characteristic function $\lambda_{t}(x, \xi)$ is real.

2.2. Uniform upper bound for characteristic functions. We begin with a uniform upper bound for characteristic functions for small $t \ll 1$.

Proposition 2.4. Let $\left(X_{t}\right)_{t \geqslant 0}$ be a Feller process with the generator $(A, D(A))$ such that $C_{c}^{\infty}\left(\mathbb{R}^{d}\right) \subset D(A)$. Then $\left.A\right|_{C_{c}^{\infty}\left(\mathbb{R}^{d}\right)}=-p(\cdot, D)$ is a pseudodifferential 
operator with symbol $p(x, \xi)$. Assume that the symbol satisfies (1.6) as well as the following sector condition: there exists some $c \in[0,1)$ such that for all $\xi \in \mathbb{R}^{d}$,

$$
\sup _{x \in \mathbb{R}^{d}}|\operatorname{Im} p(x, \xi)| \leqslant c \inf _{x \in \mathbb{R}^{d}} \operatorname{Re} p(x, \xi) .
$$

Then, for any $\xi \in \mathbb{R}^{d}$ and $\varepsilon \in(0,1-c)$, there exists some $t_{0}:=t_{0}(\xi, \varepsilon)>0$ such that for all $t \in\left[0, t_{0}\right]$,

$$
\sup _{x \in \mathbb{R}^{d}}\left|\lambda_{t}(x, \xi)\right| \leqslant \exp \left[-(1-c-\varepsilon) t \inf _{z \in \mathbb{R}^{d}} \operatorname{Re} p(z, \xi)\right] .
$$

As a direct consequence of Proposition 2.4, we get

Corollary 2.5. Let $\left(X_{t}\right)_{t \geqslant 0}$ be a Feller process with generator $(A, D(A))$ satisfying the assumptions of Proposition 2.4. Assume further that the symbol $p(x, \xi)$ is real. Then, for any $\xi \in \mathbb{R}^{d}$ and $\delta \in(0,1)$, there exists some $t_{0}:=t_{0}(\xi, \delta)>0$ such that for any $t \in\left[0, t_{0}\right]$,

$$
\sup _{x \in \mathbb{R}^{d}}\left|\lambda_{t}(x, \xi)\right| \leqslant \exp \left[-\delta t \inf _{z \in \mathbb{R}^{d}} p(z, \xi)\right] .
$$

Proof of Proposition 2.4. Fix $\xi \in \mathbb{R}^{d}$ and $\varepsilon \in(0,1-c)$. Without loss of generality, we may assume that $\inf _{z \in \mathbb{R}^{d}} \operatorname{Re} p(z, \xi)>0$ and $\xi \neq 0$; otherwise, the assertion (2.18) would be trivial.

Step 1. Denote by $P(t, x, d y)$ the transition function of the Feller process $\left(X_{t}\right)_{t \geqslant 0}$ and write $e_{\xi}(x)=e^{i\langle\xi, x\rangle}$ for $x, \xi \in \mathbb{R}^{d}$. Below we will examine the technique in the proof of Theorem 2.1 (i) in detail. Since there exists a constant $c>0$ such that $|p(x, \xi)| \leqslant c\left(1+|\xi|^{2}\right)$ for all $x, \xi \in \mathbb{R}^{d}$, the Feller operator $A$ has an extension such that $A e_{\xi}$ is well defined; cf. [31, Lemma 2.3] or Proposition 4.2 in the appendix. The assumption $p(\cdot, 0) \equiv 0$ guarantees, see [30, Theorem 5.2], that the process $\left(X_{t}\right)_{t \geqslant 0}$ is conservative. Therefore, see [31, Corollary 3.6] or Proposition 4.2, we find for $t>0$ and $x, \xi \in \mathbb{R}^{d}$,

$$
T_{t} e_{\xi}(x)=e_{\xi}(x)+\int_{0}^{t} T_{s} A e_{\xi}(x) d s .
$$

Note that, see e.g. [31, Proof of Lemma 6.3, Page 607, Lines 14-15],

$$
-p(x, \xi)=e_{-\xi}(x) A e_{\xi}(x) .
$$

Therefore,

$$
\begin{aligned}
\lambda_{t}(x, \xi) & =e_{-\xi}(x) T_{t} e_{\xi}(x) \\
& =1-e^{-i\langle\xi, x\rangle} \int_{0}^{t} T_{s}\left(p(\cdot, \xi) e^{i\langle\xi, \cdot\rangle}\right)(x) d s \\
& =1-\int_{0}^{t} \int p(y, \xi) e^{i\langle y-x, \xi\rangle} P(s, x, d y) d s .
\end{aligned}
$$

Step 2. Denote by $\operatorname{Re} z$ and $\operatorname{Im} z$ the real and imaginary parts of $z \in \mathbb{C}$. From (2.12) we get

$$
\operatorname{Re} \lambda_{t}(x, \xi)=\int \cos \langle y-x, \xi\rangle P(s, x, d y)
$$

and

$$
\operatorname{Im} \lambda_{t}(x, \xi)=\int \sin \langle y-x, \xi\rangle P(s, x, d y) .
$$


Using (2.1), we find for all $t>0$ and $x, \xi \in \mathbb{R}^{d}$,

$\operatorname{Re} \lambda_{t}(x, \xi)=1-\int_{0}^{t} \int(\cos \langle y-x, \xi\rangle \operatorname{Re} p(y, \xi)-\sin \langle y-x, \xi\rangle \operatorname{Im} p(y, \xi)) P(s, x, d y) d s$.

Thus, for every $t>0$,

$$
\begin{aligned}
\operatorname{Re} \lambda_{t}(x, \xi) & \geqslant 1-\int_{0}^{t} \int(\operatorname{Re} p(y, \xi)+|\operatorname{Im} p(y, \xi)|) P(s, x, d y) d s \\
& \geqslant 1-2 \sup _{z \in \mathbb{R}^{d}}|p(z, \xi)| t .
\end{aligned}
$$

For every $\varepsilon \in(0,1-c)$ we define $t_{1}=t_{1}(\xi, \varepsilon)>0$ by

$$
t_{1}:=\frac{\varepsilon}{8 \sup _{z \in \mathbb{R}^{d}}|p(z, \xi)|} .
$$

Then we find for all $t \in\left(0, t_{1}\right]$,

$$
\operatorname{Re} \lambda_{t}(x, \xi) \geqslant 1-\frac{\varepsilon}{4} .
$$

Set

$$
g_{1}(\xi, \varepsilon):=\frac{\varepsilon}{4|\xi|}\left[\frac{\inf _{z \in \mathbb{R}^{d}} \operatorname{Re} p(z, \xi)}{1+\sup _{z \in \mathbb{R}^{d}}|\operatorname{Im} p(z, \xi)|} \wedge 1\right]
$$

and denote by $\tau_{B(x, r)}$ the first exit time of the process from the open ball $B(x, r)$, i.e.

$$
\tau_{B(x, r)}:=\inf \left\{t>0: X_{t} \notin B(x, r)\right\} .
$$

Then we have for every $t>0$ and $x, \xi \in \mathbb{R}^{d}$,

$$
\begin{aligned}
\operatorname{Re} \lambda_{t}(x, \xi) \leqslant 1 & -\int_{0}^{t} \int_{\left\{|y-x| \leqslant g_{1}(\xi, \varepsilon)\right\}} \operatorname{Re} p(y, \xi) \cos \langle y-x, \xi\rangle P(s, x, d y) d s \\
& +\int_{0}^{t} \int_{\left\{|y-x| \leqslant g_{1}(\xi, \varepsilon)\right\}}|\operatorname{Im} p(y, \xi)||\sin \langle y-x, \xi\rangle| P(s, x, d y) d s \\
& +2 \sup _{z \in \mathbb{R}^{d}}|p(z, \xi)| \int_{0}^{t} \mathbb{P}^{x}\left(\left|X_{s}-x\right| \geqslant g_{1}(\xi, \varepsilon)\right) d s \\
\leqslant 1 & -\inf _{z \in \mathbb{R}^{d}} \operatorname{Re} p(z, \xi) \int_{0}^{t} \int_{\left\{|y-x| \leqslant g_{1}(\xi, \varepsilon)\right\}} \cos \langle y-x, \xi\rangle P(s, x, d y) d s \\
& +\sup _{z \in \mathbb{R}^{d}}|\operatorname{Im} p(z, \xi)| \int_{0}^{t} \int_{\left\{|y-x| \leqslant g_{1}(\xi, \varepsilon)\right\}}|\sin \langle y-x, \xi\rangle| P(s, x, d y) d s \\
& +2 \sup _{z \in \mathbb{R}^{d}}|p(z, \xi)| \int_{0}^{t} \mathbb{P}^{x}\left(\tau_{\left.B\left(x, g_{1}(\xi, \varepsilon)\right) \leqslant s\right) d s .}\right.
\end{aligned}
$$

In the second inequality we used that $\cos \langle y-x, \xi\rangle \geqslant 0$ on the set $\left\{|y-x| \leqslant g_{1}(\xi, \varepsilon)\right\}$ and $\left\{\left|X_{s}-x\right| \geqslant g_{1}(\xi, \varepsilon)\right\} \subset\left\{\tau_{B\left(x, g_{1}(\xi, \varepsilon)\right)} \leqslant s\right\}$.

We know from [31, Lemmas 4.1 and Lemma 5.1], see also Proposition 4.3] in the appendix for a simple self-contained proof, that for $x, \xi \in \mathbb{R}^{d}$ and $s>0$,

$$
\begin{aligned}
& \mathbb{P}^{x}\left(\tau_{\left.B\left(x, g_{1}(\xi, \varepsilon)\right) \leqslant s\right)} \leqslant c_{1} s \sup _{|y-x| \leqslant g_{1}(\xi, \varepsilon)} \sup _{|\eta| \leqslant 1 / g_{1}(\xi, \varepsilon)}|p(y, \eta)|\right. \\
& \leqslant c_{1} s \sup _{z \in \mathbb{R}^{d}} \sup _{|\eta| \leqslant 1 / g_{1}(\xi, \varepsilon)}|p(z, \eta)|
\end{aligned}
$$


for some absolute constant $c_{1}>0$. Note that on the set $\left\{|y-x| \leqslant g_{1}(\xi, \varepsilon)\right\}$,

$$
|\sin \langle y-x, \xi\rangle| \leqslant|\langle y-x, \xi\rangle| \leqslant g_{1}(\xi, \varepsilon)|\xi| .
$$

If we combine all estimates from above, we arrive at

$$
\begin{aligned}
\operatorname{Re} \lambda_{t}(x, \xi) \leqslant 1 & -\inf _{z \in \mathbb{R}^{d}} \operatorname{Re} p(z, \xi) \int_{0}^{t} \int_{\left\{|y-x| \leqslant g_{1}(\xi, \varepsilon)\right\}} \cos \langle y-x, \xi\rangle P(s, x, d y) d s \\
& +\frac{\varepsilon}{4} \inf _{z \in \mathbb{R}^{d}} \operatorname{Re} p(z, \xi) t+c_{1} \sup _{z \in \mathbb{R}^{d}}|p(z, \xi)| \sup _{z \in \mathbb{R}^{d}|\eta| \leqslant 1 / g_{1}(\xi, \varepsilon)}|p(z, \eta)| t^{2} \\
\leqslant 1 & -\inf _{z \in \mathbb{R}^{d}} \operatorname{Re} p(z, \xi) \int_{0}^{t} \int \cos \langle y-x, \xi\rangle P(s, x, d y) d s \\
& +\inf _{z \in \mathbb{R}^{d}} \operatorname{Re} p(z, \xi) \int_{0}^{t} \mathbb{P}^{x}\left(\left|X_{s}-x\right| \geqslant g_{1}(\xi, \varepsilon)\right) d s \\
& +\frac{\varepsilon}{4} \inf _{z \in \mathbb{R}^{d}} \operatorname{Re} p(z, \xi) t+c_{1} \sup _{z \in \mathbb{R}^{d}}|p(z, \xi)| \sup _{z \in \mathbb{R}^{d}|\eta| \leqslant 1 / g_{1}(\xi, \varepsilon)}|p(z, \eta)| t^{2} \\
\leqslant 1 & -\inf _{z \in \mathbb{R}^{d}} \operatorname{Re} p(z, \xi) \int_{0}^{t} \int \cos \langle y-x, \xi\rangle P(s, x, d y) d s \\
& +\frac{c_{1}}{2} \inf _{z \in \mathbb{R}^{d}} \operatorname{Re} p(z, \xi) \sup _{z \in \mathbb{R}^{d}|\eta| \leqslant 1 / g_{1}(\xi, \varepsilon)} \sup _{|p(z, \eta)| t^{2}} \\
& +\frac{\varepsilon}{4} \inf _{z \in \mathbb{R}^{d}} \operatorname{Re} p(z, \xi) t+c_{1} \sup _{z \in \mathbb{R}^{d}}|p(z, \xi)| \sup _{z \in \mathbb{R}^{d}|\eta| \leqslant 1 / g_{1}(\xi, \varepsilon)}|p(z, \eta)| t^{2} \\
=1 & -\inf _{z \in \mathbb{R}^{d}} \operatorname{Re} p(z, \xi) \int_{0}^{t} \operatorname{Re}_{s}(x, \xi) d s \\
& +\frac{\varepsilon}{4} \inf _{z \in \mathbb{R}^{d}} \operatorname{Re} p(z, \xi) t+\frac{3 c_{1}}{2} \sup _{z \in \mathbb{R}^{d}}|p(z, \xi)| \sup _{z \in \mathbb{R}^{d}} \sup _{|\eta| \leqslant 1 / g_{1}(\xi, \varepsilon)}|p(z, \eta)| t^{2} .
\end{aligned}
$$

For the third inequality we used $\left\{\left|X_{s}-x\right| \geqslant g_{1}(\xi, \varepsilon)\right\} \subset\left\{\tau_{B\left(x, g_{1}(\xi, \varepsilon)\right)} \leqslant s\right\}$ and (2.25), while the last equality follows from (2.22).

Using (2.24) we find for all $t \in\left(0, t_{1}\right]$,

$$
\begin{aligned}
\operatorname{Re} \lambda_{t}(x, \xi) \leqslant 1 & -\left(1-\frac{\varepsilon}{2}\right) \inf _{z \in \mathbb{R}^{d}} \operatorname{Re} p(z, \xi) t \\
& +\frac{3 c_{1}}{2} \sup _{z \in \mathbb{R}^{d}}|p(z, \xi)| \sup _{z \in \mathbb{R}^{d}} \sup _{|\eta| \leqslant 1 / g_{1}(\xi, \varepsilon)}|p(z, \eta)| t^{2} .
\end{aligned}
$$

Step 3. We will now consider $\operatorname{Im} \lambda_{t}(x, \xi)$. For every $t>0$ and $x, \xi \in \mathbb{R}^{d}$, we find from (2.1) that

$\operatorname{Im} \lambda_{t}(x, \xi)=-\int_{0}^{t} \int(\cos \langle y-x, \xi\rangle \operatorname{Im} p(y, \xi)+\sin \langle y-x, \xi\rangle \operatorname{Re} p(y, \xi)) P(s, x, d y) d s$.

Therefore, for each $t>0$,

$$
\left|\operatorname{Im} \lambda_{t}(x, \xi)\right| \leqslant \sup _{z \in \mathbb{R}^{d}}|\operatorname{Im} p(z, \xi)| t+\int_{0}^{t} \int \operatorname{Re} p(y, \xi)|\sin \langle y-x, \xi\rangle| P(s, x, d y) d s .
$$

Set

$$
g_{2}(\xi, \varepsilon):=\frac{\varepsilon}{4|\xi|} \frac{\inf _{z \in \mathbb{R}^{d}} \operatorname{Re} p(z, \xi)}{\sup _{z \in \mathbb{R}^{d}} \operatorname{Re} p(z, \xi)}
$$


Then, similar to the reasoning in Step 2, we see

$$
\begin{aligned}
\left|\operatorname{Im} \lambda_{t}(x, \xi)\right| \leqslant & \sup _{z \in \mathbb{R}^{d}}|\operatorname{Im} p(z, \xi)| t \\
& +\sup _{z \in \mathbb{R}^{d}} \operatorname{Re} p(z, \xi) \int_{0}^{t} \int_{\left\{|y-x| \leqslant g_{2}(\xi, \varepsilon)\right\}}|\sin \langle y-x, \xi\rangle| P(s, x, d y) d s \\
& +\sup _{z \in \mathbb{R}^{d}} \operatorname{Re} p(z, \xi) \int_{0}^{t} \mathbb{P}^{x}\left(\left|X_{s}-x\right| \geqslant g_{2}(\xi, \varepsilon)\right) d s \\
\leqslant & \sup _{z \in \mathbb{R}^{d}}|\operatorname{Im} p(z, \xi)| t+\frac{\varepsilon}{4} \inf _{z \in \mathbb{R}^{d}} \operatorname{Re} p(z, \xi) t \\
& +\frac{c_{1}}{2} \sup _{z \in \mathbb{R}^{d}}|p(z, \xi)| \sup _{z \in \mathbb{R}^{d}} \sup _{|\eta| \leqslant 1 / g_{2}(\xi, \varepsilon)}|p(z, \eta)| t^{2} .
\end{aligned}
$$

This along with (2.24) and (2.26) yields for all $t \in\left(0, t_{1}\right]$,

$$
\begin{aligned}
\left|\lambda_{t}(x, \xi)\right| \leqslant & \left|\operatorname{Re} \lambda_{t}(x, \xi)\right|+\left|\operatorname{Im} \lambda_{t}(x, \xi)\right| \\
= & \operatorname{Re} \lambda_{t}(x, \xi)+\left|\operatorname{Im} \lambda_{t}(x, \xi)\right| \\
\leqslant & 1-\left[\left(1-\frac{3 \varepsilon}{4}\right) \inf _{z \in \mathbb{R}^{d}} \operatorname{Re} p(z, \xi)-\sup _{z \in \mathbb{R}^{d}}|\operatorname{Im} p(z, \xi)|\right] t \\
& +\frac{c_{1}}{2} \sup _{z \in \mathbb{R}^{d}}|p(z, \xi)|\left[3 \sup _{z \in \mathbb{R}^{d}} \sup _{|\eta| \leqslant 1 / g_{1}(\xi, \varepsilon)}|p(z, \eta)|\right. \\
& \left.+\sup _{z \in \mathbb{R}^{d}|\eta| \leqslant 1 / g_{2}(\xi, \varepsilon)}|p(z, \eta)|\right] t^{2} .
\end{aligned}
$$

Now set

$$
S:=2 c_{1} \sup _{z \in \mathbb{R}^{d}}|p(z, \xi)|\left[3 \sup _{z \in \mathbb{R}^{d}} \sup _{|\eta| \leqslant 1 / g_{1}(\xi, \varepsilon)}|p(z, \eta)|+\sup _{z \in \mathbb{R}^{d}} \sup _{|\eta| \leqslant 1 / g_{2}(\xi, \varepsilon)}|p(z, \eta)|\right]
$$

and define $t_{2}=t_{2}(\varepsilon, \xi)$ by

$$
t_{2}:=t_{1} \wedge \frac{\varepsilon \inf _{z \in \mathbb{R}^{d}} \operatorname{Re} p(z, \xi)}{S} .
$$

Then we obtain for all $t \in\left(0, t_{2}\right]$,

$$
\left|\lambda_{t}(x, \xi)\right| \leqslant 1-\left[(1-\varepsilon) \inf _{z \in \mathbb{R}^{d}} \operatorname{Re} p(z, \xi)-\sup _{z \in \mathbb{R}^{d}}|\operatorname{Im} p(z, \xi)|\right] t .
$$

Because of the sector condition (2.17), we see

$$
\begin{aligned}
\left|\lambda_{t}(x, \xi)\right| & \leqslant 1-(1-c-\varepsilon) t \inf _{z \in \mathbb{R}^{d}} \operatorname{Re} p(z, \xi) \\
& \leqslant \exp \left[-(1-c-\varepsilon) t \inf _{z \in \mathbb{R}^{d}} \operatorname{Re} p(z, \xi)\right],
\end{aligned}
$$

where the last estimate follows from the elementary inequality $1-r \leqslant e^{-r}$ for $r \in \mathbb{R}$. In particular, for any $t \in\left(0, t_{2}\right]$,

$$
\sup _{x \in \mathbb{R}^{d}}\left|\lambda_{t}(x, \xi)\right| \leqslant \exp \left[-(1-c-\varepsilon) t \inf _{z \in \mathbb{R}^{d}} \operatorname{Re} p(z, \xi)\right],
$$

which is the required assertion by taking $t_{0}=t_{2}$. 
Remark 2.6. (i) Note that $t_{2}(\varepsilon, \xi) \rightarrow 0$ as $\varepsilon \rightarrow 0$, which means that the approach above fails for $\varepsilon=0$. Therefore, Proposition 2.4 will, in general, not hold with $\varepsilon=0$ nor can we expect Corollary 2.5 to be true if $\delta=1$.

(ii) A variant of our approach yields a uniform lower bound for characteristic functions for small $t$. More precisely: Let $\left(X_{t}\right)_{t \geqslant 0}$ be a Feller process with the generator $(A, D(A))$ such that $C_{c}^{\infty}\left(\mathbb{R}^{d}\right) \subset D(A)$. Then $\left.A\right|_{C_{c}^{\infty}\left(\mathbb{R}^{d}\right)}=-p(\cdot, D)$ is a pseudodifferential operator with symbol $p(x, \xi)$. Assume that the symbol satisfies (1.6). Then for any $\varepsilon>0$ and $\xi \in \mathbb{R}^{d}$, there exists some $t_{0}=t_{0}(\varepsilon, \xi)>0$ such that for any $t \in\left(0, t_{0}\right]$,

$$
\inf _{x \in \mathbb{R}^{d}}\left|\lambda_{t}(x, \xi)\right| \geqslant \exp \left(-(1+\varepsilon) t \sup _{z \in \mathbb{R}^{d}}|p(z, \xi)|\right) .
$$

(iii) From the pointwise estimate (2.15), one can get that for any $\xi \in \mathbb{R}^{d}$, there exists some $t_{0}:=t_{0}(\xi)>0$ such that for all $t \in\left[0, t_{0}\right]$,

$$
\sup _{x \in \mathbb{R}^{d}}\left|\lambda_{t}(x, \xi)\right| \leqslant e^{-t \inf _{x \in \mathbb{R}^{d}} \operatorname{Re} p(x, \xi)}+C(\xi, 1) t .
$$

Although the remainder term $C(\xi, 1)$ in (2.15) is well known, see [28, Lemma 2] for details, we were not able to derive the assertion (2.18) from this estimate.

Our main result in this subsection is the following uniform upper bound of the characteristic function, which is just Theorem 1.1 in Section 1.

Theorem 2.7. Let $\left(X_{t}\right)_{t \geqslant 0}$ be a Feller process with the generator $(A, D(A))$ such that $C_{c}^{\infty}\left(\mathbb{R}^{d}\right) \subset D(A)$; i.e., $\left.A\right|_{C_{c}^{\infty}\left(\mathbb{R}^{d}\right)}=-p(\cdot, D)$ is a pseudodifferential operator with symbol $p(x, \xi)$. For all $x \in \mathbb{R}^{d}$ and $t \geqslant 0$, let $\lambda_{t}(x, \xi)$ be the characteristic function of $\left(X_{t}\right)_{t \geqslant 0}$ given by (2.12). Assume that the symbol satisfies (1.6). Then, for all $t \geqslant 0$ and $\xi \in \mathbb{R}^{d}$,

$$
\sup _{x \in \mathbb{R}^{d}}\left|\lambda_{t}(x, \xi)\right| \leqslant \exp \left[-\frac{t}{16} \inf _{z \in \mathbb{R}^{d}} \operatorname{Re} p(z, 2 \xi)\right] .
$$

Proof of Step 1. First we assume that the characteristic function $\lambda_{t}(x, \xi)$ is real for every $t \geqslant 0$ and every $x, \xi \in \mathbb{R}^{d}$. Then, by Theorem 2.1 (ii), the corresponding symbol $p(x, \xi)$ is also real. On the other hand, applying Corollary 2.5 with $\delta=1 / 2$ yields that there exists some $t_{0}:=t_{0}(\xi)>0$ such that for all $t \in\left(0, t_{0}\right]$,

$$
\sup _{x \in \mathbb{R}^{d}}\left|\lambda_{t}(x, \xi)\right| \leqslant \exp \left[-\frac{1}{2} t \inf _{z \in \mathbb{R}^{d}} p(z, \xi)\right] .
$$

Since $\sqrt{p(x, \cdot)}$ is subadditive, i.e. $\sqrt{p\left(x, \xi_{1}+\xi_{2}\right)} \leqslant \sqrt{p\left(x, \xi_{1}\right)}+\sqrt{p\left(x, \xi_{2}\right)}$ for all $x, \xi_{1}, \xi_{2} \in \mathbb{R}^{d}$, we see

Thus, (2.28) leads to

$$
\inf _{z \in \mathbb{R}^{d}} p(z, 2 \xi) \leqslant 4 \inf _{z \in \mathbb{R}^{d}} p(z, \xi)
$$

$$
\sup _{x \in \mathbb{R}^{d}}\left|\lambda_{t}(x, \xi)\right| \leqslant \exp \left[-\frac{1}{8} t \inf _{z \in \mathbb{R}^{d}} p(z, 2 \xi)\right] .
$$

For every $t>0$ we can choose some $m:=m(\xi) \in \mathbb{N}$ such that $\frac{t}{m} \in\left(0, t_{3}\right]$, where

$$
t_{3}=t_{0} \wedge \frac{2}{\inf _{z \in \mathbb{R}^{d}} p(z, 2 \xi)} .
$$


We will prove by induction that for any $k=1,2, \ldots, m$,

$$
\sup _{x \in \mathbb{R}^{d}}\left|\lambda_{k \frac{t}{m}}(x, \xi)\right| \leqslant \exp \left[-\frac{k}{16} \frac{t}{m} \inf _{z \in \mathbb{R}^{d}} p(z, 2 \xi)\right] .
$$

First, according to (2.29), we know that (2.30) holds with $k=1$. Assume that (2.30) is satisfied with $k=j$. Then, for $k=j+1$, by the Markov property and the fact that the characteristic function $\lambda_{t}(x, \xi)$ is real for any $t \geqslant 0$ and $x, \xi \in \mathbb{R}^{d}$, we have

$$
\begin{aligned}
\left|\lambda_{(j+1) t / m}(x, \xi)\right| & =\left|\mathbb{E}^{x}\left(e^{i\left\langle X_{(j+1) t / m}-x, \xi\right\rangle}\right)\right| \\
& =\left|\mathbb{E}^{x}\left(e^{i\left\langle X_{t / m}-x, \xi\right\rangle} \mathbb{E}^{X_{t / m}}\left(e^{i\left\langle X_{j t / m}-x, \xi\right\rangle}\right)\right)\right| \\
& =\left|\mathbb{E}^{x}\left(e^{i\left\langle X_{t / m}-x, \xi\right\rangle} \lambda_{j t / m}\left(X_{t / m}, \xi\right)\right)\right| \\
& =\left|\mathbb{E}^{x}\left(\cos \left\langle X_{t / m}-x, \xi\right\rangle \lambda_{j t / m}\left(X_{t / m}, \xi\right)\right)\right| \\
& \leqslant\left[\mathbb{E}^{x}\left(\cos ^{2}\left\langle X_{t / m}-x, \xi\right\rangle\right)\right]^{1 / 2}\left[\mathbb{E}^{x}\left(\lambda_{j t / m}^{2}\left(X_{t / m}, \xi\right)\right)\right]^{1 / 2} \\
& \leqslant\left[\mathbb{E}^{x}\left(\cos ^{2}\left\langle X_{t / m}-x, \xi\right\rangle\right)\right]^{1 / 2} \sup _{x \in \mathbb{R}^{d}}\left|\lambda_{j t / m}(x, \xi)\right| \\
& \leqslant\left[\frac{1+\mathbb{E}^{x}\left(\cos \left\langle X_{t / m}-x, 2 \xi\right\rangle\right)}{2}\right]^{1 / 2} \exp \left[-\frac{j}{16} \frac{t}{m} \inf _{z \in \mathbb{R}^{d}} p(z, 2 \xi)\right] .
\end{aligned}
$$

The first inequality follows from the Cauchy-Schwarz inequality, and in the last inequality we have used the induction hypothesis and the fact that

$$
\cos ^{2} \theta=\frac{1}{2}(1+\cos (2 \theta)), \quad \theta \in \mathbb{R} .
$$

Therefore,

$$
\sup _{x \in \mathbb{R}^{d}}\left|\lambda_{(j+1) \frac{t}{m}}(x, \xi)\right| \leqslant \sup _{x \in \mathbb{R}^{d}}\left[\frac{1+\mathbb{E}^{x}\left(\cos \left\langle X_{t / m}-x, 2 \xi\right\rangle\right)}{2}\right]^{\frac{1}{2}} \exp \left[-\frac{j}{16} \frac{t}{m} \inf _{z \in \mathbb{R}^{d}} p(z, 2 \xi)\right] \text {. }
$$

For any $x \in \mathbb{R}^{d}$ we can use (2.28) and the assumptions that $\lambda_{t}(x, \xi)$ is real and $\frac{t}{2 m} \inf _{z \in \mathbb{R}^{d}} p(z, 2 \xi) \leqslant 1$ to deduce

$$
\begin{aligned}
\frac{1+\mathbb{E}^{x}\left(\cos \left\langle X_{t / m}-x, 2 \xi\right\rangle\right)}{2} & \\
& =\frac{1+\lambda_{t / m}(x, 2 \xi)}{2} \\
& \leqslant \frac{1+\left|\lambda_{t / m}(x, 2 \xi)\right|}{2} \\
& \leqslant \frac{1+\exp \left[-\frac{t}{2 m} \inf _{z \in \mathbb{R}^{d}} p(z, 2 \xi)\right]}{2} \\
& \leqslant \frac{1+1-\left[\frac{t}{2 m} \inf _{z \in \mathbb{R}^{d}} p(z, 2 \xi)\right]+\frac{1}{2}\left[\frac{t}{2 m} \inf _{z \in \mathbb{R}^{d}} p(z, 2 \xi)\right]^{2}}{2}
\end{aligned}
$$




$$
\begin{aligned}
& \leqslant 1-\frac{\frac{t}{m} \inf _{z \in \mathbb{R}^{d}} p(z, 2 \xi)}{8} \\
& \leqslant \exp \left[-\frac{\frac{t}{m} \inf _{z \in \mathbb{R}^{d}} p(z, 2 \xi)}{8}\right],
\end{aligned}
$$

where the third and the last inequality follow from the elementary estimates

$$
1-r \leqslant e^{-r} \leqslant 1-r+r^{2} / 2, \quad r \geqslant 0 .
$$

Thus, we get

$$
\sup _{x \in \mathbb{R}^{d}}\left[\frac{1+\mathbb{E}^{x}\left(\cos \left\langle X_{t / m}-x, 2 \xi\right\rangle\right)}{2}\right]^{1 / 2} \leqslant \exp \left[-\frac{\frac{t}{m} \inf _{z \in \mathbb{R}^{d}} p(z, 2 \xi)}{16}\right]
$$

and the induction step is complete.

Taking $k=m$ in (2.30) we find, in particular, for all $t>0$,

$$
\sup _{x \in \mathbb{R}^{d}}\left|\lambda_{t}(x, \xi)\right| \leqslant \exp \left[-\frac{t}{16} \inf _{z \in \mathbb{R}^{d}} p(z, 2 \xi)\right]
$$

Step 2. Now we consider the general case where $\lambda_{t}(x, \xi)$ is not necessarily real. Using a local symmetrization technique we can reduce the general case to the situation treated in Step 1. Let $\left(X_{t}\right)_{t \geqslant 0}$ be a Feller process with the generator $(A, D(A))$ and the semigroup $\left(T_{t}\right)_{t \geqslant 0}$ such that $C_{c}^{\infty}\left(\mathbb{R}^{d}\right) \subset D(A)$ and $\left.A\right|_{C_{c}^{\infty}\left(\mathbb{R}^{d}\right)}=-p(\cdot, D)$ is a pseudodifferential operator with symbol $p(x, \xi)$. Denote by $\lambda_{t}(x, \xi)$ the characteristic function of $X_{t}-x$ under $\mathbb{P}^{x}$.

Construct on the same probability space a stochastic process $\left(X_{t}^{*}\right)_{t \geqslant 0}$ such that $X_{0}^{*}=X_{0}$ and $\left(X_{t}^{*}\right)_{t>0}$ is an independent copy of $\left(X_{t}\right)_{t>0}$, and define a further process $\left(\widetilde{X}_{t}\right)_{t \geqslant 0}$ on $\mathbb{R}^{d}$ by $\widetilde{X}_{t}=2 X_{0}^{*}-X_{t}^{*}, t \geqslant 0$. Clearly, the process $\left(\widetilde{X}_{t}\right)_{t>0}$ is independent of $\left(X_{t}\right)_{t>0}$ but it has the same initial distribution, i.e. $\widetilde{X}_{0} \sim X_{0}$. From the proof of Theorem 2.1 (iii) we see that $\left(\widetilde{X}_{t}\right)_{t \geqslant 0}$ is a Feller process with the generator $(\widetilde{A}, D(\widetilde{A}))$ and the semigroup $\left(\widetilde{T}_{t}\right)_{t \geqslant 0}$ such that $C_{c}^{\infty}\left(\mathbb{R}^{d}\right) \subset D(\widetilde{A})$, and $\left.\widetilde{A}\right|_{c} ^{\infty}\left(\mathbb{R}^{d}\right)=-\widetilde{p}(\cdot, D)$ is a pseudodifferential operator with symbol $\widetilde{p}(x, \xi)=$ $p(x,-\xi)$. Moreover, the characteristic function of $\left(\tilde{X}_{t}\right)_{t \geqslant 0}$ is $\widetilde{\lambda}_{t}(x, \xi)=\lambda_{t}(x,-\xi)$ for every $t \geqslant 0$ and $x \in \mathbb{R}^{d}$.

For every $t \geqslant 0$ we define the local symmetrization $X_{t}^{S}=\frac{1}{2}\left(X_{t}+\widetilde{X}_{t}\right)$. Lemma 2.8 below shows that the local symmetrization $\left(X_{t}^{S}\right)_{t \geqslant 0}$ is a Feller process with the generator $\left(A^{S}, D\left(A^{S}\right)\right)$ such that $C_{c}^{\infty}\left(\mathbb{R}^{d}\right) \subset D\left(A^{S}\right)$, and $\left.A^{S}\right|_{C_{c}^{\infty}\left(\mathbb{R}^{d}\right)}=-p^{S}(\cdot, D)$ is a pseudodifferential operator with symbol $2 \operatorname{Re} p(x, \xi / 2)$; moreover, the characteristic function of $\left(X_{t}^{S}\right)_{t \geqslant 0}$ is $\left|\lambda_{t}(x, \xi / 2)\right|^{2}$.

We can now apply the conclusion of Step 1, in particular (2.31), to the process $\left(X_{t}^{S}\right)_{t \geqslant 0}$; we obtain that for any $t>0$,

$$
\sup _{x \in \mathbb{R}^{d}}\left|\lambda_{t}(x, \xi / 2)\right|^{2} \leqslant \exp \left[-\frac{t}{8} \inf _{z \in \mathbb{R}^{d}} \operatorname{Re} p(z, \xi)\right]
$$


That is,

$$
\sup _{x \in \mathbb{R}^{d}}\left|\lambda_{t}(x, \xi)\right|^{2} \leqslant \exp \left[-\frac{t}{8} \inf _{z \in \mathbb{R}^{d}} \operatorname{Re} p(z, 2 \xi)\right]
$$

which is what we have claimed.

Lemma 2.8 (Local Symmetrization). Let $\left(X_{t}\right)_{t \geqslant 0}$ be a Feller process with generator $(A, D(A))$ such that $C_{c}^{\infty}\left(\mathbb{R}^{d}\right) \subset D(A)$, i.e. $\left.A\right|_{C_{c}^{\infty}\left(\mathbb{R}^{d}\right)}=-p(\cdot, D)$. Denote by $\left(X_{t}^{*}\right)_{t \geqslant 0}$ an independent copy of $\left(X_{t}\right)_{t \geqslant 0}$, set $\tilde{X}_{t}:=2 X_{0}^{*}-X_{t}^{*}$ and let $\left(X_{t}^{S}\right)_{t \geqslant 0}$ be the local symmetrization of $\left(X_{t}\right)_{t \geqslant 0}$, i.e. for any $t \geqslant 0, X_{t}^{S}=\frac{1}{2}\left(X_{t}+\widetilde{X}_{t}\right)$. Then, $\left(X_{t}^{S}\right)_{t \geqslant 0}$ is a Feller process with the generator $\left(A^{S}, D\left(A^{S}\right)\right)$ such that

(i) $C_{c}^{\infty}\left(\mathbb{R}^{d}\right) \subset D\left(A^{S}\right)$, and $\left.A^{S}\right|_{C_{c}^{\infty}\left(\mathbb{R}^{d}\right)}=-p^{S}(\cdot, D)$ is a pseudodifferential operator with symbol $p^{S}(x, \xi)=2 \operatorname{Re} p(x, \xi / 2)$;

(ii) the characteristic function of $\left(X_{t}^{S}\right)_{t \geqslant 0}$ is $\left|\lambda_{t}(x, \xi / 2)\right|^{2}$ for every $t \geqslant 0$ and $x \in \mathbb{R}^{d}$.

Proof. Clearly, $\left(X_{t}^{S}\right)_{t \geqslant 0}$ is a strong Markov process. Denote by $\left(T_{t}^{S}\right)_{t \geqslant 0}$ the semigroup of $\left(X_{t}^{S}\right)_{t \geqslant 0}$. Since $\left(X_{t}\right)_{t>0}$ and $(\tilde{X})_{t>0}$ are independent with $X_{0} \sim \widetilde{X}_{0}$ we find that for all $u \in B_{b}\left(\mathbb{R}^{d}\right)$ (the set of bounded measurable functions on $\mathbb{R}^{d}$ ), $x \in \mathbb{R}^{d}$ and $t \geqslant 0$,

$$
T_{t}^{S} u(x)=\int u(z) P^{S}(t, x, d z)=\iint u\left(\frac{z_{1}+z_{2}}{2}\right) P\left(t, x, d z_{1}\right) \widetilde{P}\left(t, x, d z_{2}\right),
$$

where $P(t, x, d y), \widetilde{P}(t, x, d y)$ and $P^{S}(t, x, d y)$ are the transition functions of $\left(X_{t}\right)_{t \geqslant 0}$, $\left(\widetilde{X}_{t}\right)_{t \geqslant 0}$ and $\left(X_{t}^{S}\right)_{t \geqslant 0}$, respectively.

As mentioned above, since $p(\cdot, 0) \equiv 0$, 30, Theorem 5.2] shows that the processes $\left(X_{t}\right)_{t \geqslant 0}$ and $\left(\widetilde{X}_{t}\right)_{t \geqslant 0}$ are conservative. Thus, according to Proposition 4.5 (i) in the appendix, both semigroups $\left(T_{t}\right)_{t \geqslant 0}$ and $\left(\widetilde{T}_{t}\right)_{t \geqslant 0}$ are $C_{b}$-Feller semigroups; i.e., they map the space $C_{b}\left(\mathbb{R}^{d}\right)$ of bounded continuous functions on $\mathbb{R}^{d}$ into itself. This along with (2.32) yields the $C_{b}$-Feller property of $\left(T_{t}^{S}\right)_{t \geqslant 0}$. Indeed, for any fixed $x_{0} \in \mathbb{R}^{d}$ and $t>0$, due to the $C_{b}$-Feller property of $\left(T_{t}\right)_{t \geqslant 0}$ and $\left(\widetilde{T}_{t}\right)_{t \geqslant 0}$, we know that the probability measures $P(t, x, d z)$ and $\widetilde{P}(t, x, d z)$ converge weakly to $P\left(t, x_{0}, d z\right)$ and $\widetilde{P}(t, x, d z)$ respectively, as $x$ tends to $x_{0}$. Thus, the convolution of $P(t, x, d z)$ and $\widetilde{P}(t, x, d z)$ converges weakly to the convolution of $P\left(t, x_{0}, d z\right)$ and $\widetilde{P}\left(t, x_{0}, d z\right)$ as $x$ tends to $x_{0}$. That is, for any $u \in C_{b}\left(\mathbb{R}^{d}\right)$,

$$
\lim _{x \rightarrow x_{0}} \iint u\left(z_{1}+z_{2}\right) P\left(t, x, d z_{1}\right) \widetilde{P}\left(t, x, d z_{2}\right)=\iint u\left(z_{1}+z_{2}\right) P\left(t, x_{0}, d z_{1}\right) \widetilde{P}\left(t, x_{0}, d z_{2}\right) .
$$

This immediately yields the $C_{b}$-Feller property of $\left(T_{t}^{S}\right)_{t \geqslant 0}$.

On the other hand, let $\left(A_{w}^{S}, D\left(A_{w}^{S}\right)\right)$ be the weak infinitesimal operator of the process $\left(X_{t}^{S}\right)_{t \geqslant 0}$. Again from the proof of Theorem 2.1 (iii) we deduce that $C_{c}^{\infty}\left(\mathbb{R}^{d}\right) \subset$ $D\left(A_{w}^{S}\right)$, and $\left.A_{w}^{S}\right|_{C_{c}^{\infty}\left(\mathbb{R}^{d}\right)}=-p^{S}(\cdot, D)$ is a pseudodifferential operator with symbol $p^{S}(x, \xi)=2 \operatorname{Re} p(x, \xi / 2)$. Indeed, for any $u \in C_{c}^{\infty}\left(\mathbb{R}^{d}\right)$ and $x \in \mathbb{R}^{d}$, using (2.32), the Fourier transform and the Fubini theorem, we get

$$
\begin{aligned}
& \lim _{t \rightarrow 0} \frac{T_{t}^{S} u(x)-u(x)}{t} \\
& =\lim _{t \rightarrow 0} \frac{1}{t}\left(\iint u\left(\frac{z_{1}+z_{2}}{2}\right) P\left(t, x, d z_{1}\right) \widetilde{P}\left(t, x, d z_{2}\right)-u(x)\right)
\end{aligned}
$$




$$
\begin{aligned}
& =\lim _{t \rightarrow 0} \frac{1}{t}\left(2^{d} \iint e^{i\left\langle\xi, x+z_{2}\right\rangle} \lambda_{t}(x, \xi) \widehat{u}(2 \xi) d \xi \widetilde{P}\left(t, x, d z_{2}\right)-2^{d} \int e^{i\langle 2 \xi, x\rangle} \widehat{u}(2 \xi) d \xi\right) \\
& =\lim _{t \rightarrow 0} \frac{1}{t}\left(2^{d} \int e^{i\langle\xi, 2 x\rangle} \lambda_{t}(x, \xi) \widehat{u}(2 \xi) d \xi \int e^{i\left\langle\xi, z_{2}-x\right\rangle} \widetilde{P}\left(t, x, d z_{2}\right)\right. \\
& \left.\quad-2^{d} \int e^{i\langle 2 \xi, x\rangle} \widehat{u}(2 \xi) d \xi\right) \\
& =\lim _{t \rightarrow 0} \frac{1}{t}\left(2^{d} \int e^{i\langle\xi, 2 x\rangle} \lambda_{t}(x, \xi) \lambda_{t}(x,-\xi) \widehat{u}(2 \xi) d \xi-2^{d} \int e^{i\langle 2 \xi, x\rangle} \widehat{u}(2 \xi) d \xi\right) \\
& =\lim _{t \rightarrow 0} 2^{d}\left(\int e^{i\langle 2 \xi, x\rangle} \frac{\left|\lambda_{t}(x, \xi)\right|^{2}-1}{t} \widehat{u}(2 \xi) d \xi\right) \\
& =-2^{d}\left(\int e^{i\langle 2 \xi, x\rangle}(p(x, \xi)+p(x,-\xi)) \widehat{u}(2 \xi) d \xi\right) \\
& =-\int e^{i\langle\xi, x\rangle}(2 \operatorname{Re} p(x, \xi / 2)) \widehat{u}(\xi) d \xi \\
& =-p^{S}(x, D) u(x) .
\end{aligned}
$$

The second and the fourth equalities follow from the fact that the characteristic functions corresponding to $P(t, x, d y)$ and $\widetilde{P}(t, x, d y)$ are $\lambda_{t}(x, \xi)$ and $\widetilde{\lambda}_{t}(x, \xi)=$ $\lambda_{t}(x,-\xi)$, respectively. In the third equality from below we used Theorem 2.1 (i) and the dominated convergence theorem. Therefore, the weak infinitesimal operator of $\left(T_{t}^{S}\right)_{t \geqslant 0}$ on $C_{c}^{\infty}\left(\mathbb{R}^{d}\right)$ is just $-p^{S}(\cdot, D)$. According to 9 , Chapter I, (1.49), Page 40], $\left(\left(X_{t}^{S}\right)_{t \geqslant 0},\left(\mathbb{P}^{x}\right)_{x \in \mathbb{R}^{d}}\right)$ is the solution to the martingale problem for $\left(-p^{S}(\cdot, D), C_{c}^{\infty}\left(\mathbb{R}^{d}\right)\right)$.

Furthermore, according to Lemma 2.3 , we get

$$
\varlimsup_{r \rightarrow \infty} \sup _{x \in \mathbb{R}^{d}} \sup _{|\xi| \leqslant 1 / r} \operatorname{Re} p(x, \xi / 2)=0
$$

Hence, Lemma 2.3 and Proposition 4.4 in the appendix finally imply that $\left(T_{t}^{S}\right)_{t \geqslant 0}$ is a Feller semigroup, and so $\left(X_{t}^{S}\right)_{t \geqslant 0}$ is a Feller process.

Let $\left(A^{S}, D\left(A^{S}\right)\right)$ be the Feller generator of $\left(X_{t}^{S}\right)_{t \geqslant 0}$. According to [27, Lemma 31.7, Page 209] and the conclusion above, $C_{c}^{\infty}\left(\mathbb{R}^{d}\right) \subset D\left(A^{S}\right)$ and $\left.A^{S}\right|_{C_{c}^{\infty}\left(\mathbb{R}^{d}\right)}=$ $-p^{S}(\cdot, D)$. Again by the independence of $\left(X_{t}\right)_{t>0}$ and $(\tilde{X})_{t>0}$ and the fact that $X_{0} \sim \widetilde{X}_{0}$ we see that for any $t>0$ the characteristic function of $\left(X_{t}^{S}\right)_{t \geqslant 0}$ is given by

$$
\begin{aligned}
\lambda_{t}^{S}(x, \xi) & =\mathbb{E}^{x}\left(e^{i\left\langle X_{t}^{S}-x, \xi\right\rangle}\right) \\
& =\mathbb{E}^{x}\left(e^{i\left\langle\left(X_{t}-x\right) / 2, \xi\right\rangle} \times e^{i\left\langle\left(\widetilde{X}_{t}-x\right) / 2, \xi\right\rangle}\right) \\
& =\mathbb{E}^{x}\left(e^{i\left\langle X_{t}-x, \xi / 2\right\rangle} \times e^{i\left\langle\widetilde{X}_{t}-x, \xi / 2\right\rangle}\right) \\
& =\mathbb{E}^{x}\left(e^{i\left\langle X_{t}-x, \xi / 2\right\rangle}\right) \mathbb{E}^{x}\left(e^{i\left\langle\widetilde{X}_{t}-x, \xi / 2\right\rangle}\right) \\
& =\left|\lambda_{t}(x, \xi / 2)\right|^{2}
\end{aligned}
$$

Together with Theorem 2.1 (i) this also shows that the symbol of the process $\left(X_{t}^{S}\right)_{t \geqslant 0}$ is $2 \operatorname{Re} p(x, \xi / 2)$. 


\section{Proof of Theorem 1.2 And some Applichtions}

\subsection{Proof of Theorem 1.2.}

Proof. (i) For any $t>0$,

$$
\begin{aligned}
\left\|T_{t}\right\|_{1 \rightarrow \infty} & :=\sup _{u \in L^{1}(d x),\|u\|_{1}=1}\left\|T_{t} u\right\|_{\infty} \\
& =\sup _{u \in C_{c}^{\infty}\left(\mathbb{R}^{d}\right),\|u\|_{1}=1}\left\|T_{t} u\right\|_{\infty} \\
& =\sup _{u \in C_{c}^{\infty}\left(\mathbb{R}^{d}\right),\|u\|_{1}=1} \sup _{x \in \mathbb{R}^{d}}\left|\int e^{i\langle x, \xi\rangle} \hat{u}(\xi) \lambda_{t}(x, \xi) d \xi\right| \\
& \leqslant \sup _{u \in C_{c}^{\infty}\left(\mathbb{R}^{d}\right),\|u\|_{1}=1} \sup _{x \in \mathbb{R}^{d}} \int\left|\hat{u}(\xi) \| \lambda_{t}(x, \xi)\right| d \xi \\
& \leqslant(2 \pi)^{-d} \sup _{x \in \mathbb{R}^{d}} \int\left|\lambda_{t}(x, \xi)\right| d \xi
\end{aligned}
$$

where we have used that for all $\xi \in \mathbb{R}^{d},|\hat{u}(\xi)| \leqslant(2 \pi)^{-d}\|u\|_{1}$. By assumption (1.8) and Theorem 2.4 .

$$
\left\|T_{t}\right\|_{1 \rightarrow \infty} \leqslant(2 \pi)^{-d} \int \exp \left(-\frac{t}{16} \inf _{z \in \mathbb{R}^{d}} \operatorname{Re} p(z, 2 \xi)\right) d \xi<\infty,
$$

which yields the ultracontractivity of the Feller semigroup. Now we can get the existence of the transition density and the strong Feller property of the semigroup from [38, Proposition 3.3.11] and [34, Corollary 2.2], respectively.

(ii) The assertion follows essentially from [39, Theorem 2.2] and Theorem 2.4, For the reader's convenience we repeat the relevant part of the argument from 39 , Theorem 2.2]. For $x=\left(x_{1}, \ldots, x_{d}\right)$ and $r>0$, write

$$
Q(x, r):=\left\{z=\left(z_{1}, \ldots, z_{d}\right) \in \mathbb{R}^{d}:\left|z_{j}-x_{j}\right| \leqslant r \text { for } 1 \leqslant j \leqslant d\right\} .
$$

For any $x \in \mathbb{R}^{d}$ and $r>0$, define

$$
g(y)=g_{x}(y):= \begin{cases}r^{2 d}, & \text { if } y=x \\ \prod_{j=1}^{d}\left(\frac{\sin r\left(y_{j}-x_{j}\right)}{y_{j}-x_{j}}\right)^{2}, & \text { if } y \neq x .\end{cases}
$$

If $f_{j}: \mathbb{R} \rightarrow \mathbb{R}, j=1, \ldots, d$, are functions we denote by $\bigotimes_{j=1}^{d} f_{j}(x):=\prod_{j=1}^{n} f_{j}\left(x_{j}\right)$ their tensor product. Notice that $\bigotimes_{j=1}^{d} f_{j}: \mathbb{R}^{d} \rightarrow \mathbb{R}$. Then, $g \in B_{b}\left(\mathbb{R}^{d}\right) \cap L^{1}\left(\mathbb{R}^{d}\right)$ and

$$
\hat{g}(\xi)=(8 \pi)^{-d} e^{-i\langle x, \xi\rangle}\left(\bigotimes_{j=1}^{d} \mathbb{1}_{[-r, r]} * \bigotimes_{j=1}^{d} \mathbb{1}_{[-r, r]}\right)(\xi) ;
$$

cf. [18, Table 3.5.19, Page 117, Vol. 1]. In particular, $\hat{g} \in L^{1}\left(\mathbb{R}^{d}\right)$. According to the proof of [17, Theorem 1.1], see also [17, Remark (B), Page 65], (2.13) holds for the test function $g$. That is,

$$
T_{t} g(x)=\int e^{i\langle x, \xi\rangle} \hat{g}(\xi) \lambda_{t}(x, \xi) d \xi .
$$


Since $\left|\frac{\sin r}{r}\right| \geqslant 1 / 2$ for $|r| \leqslant \pi / 3$, we know that $g(y) \geqslant\left(4^{-1} r\right)^{2 d}$ for all $y \in$ $Q(x, \pi /(3 r))$. For $s>0$, write $X_{s}=\left(X_{s}^{1}, \ldots, X_{s}^{d}\right)$. By monotone convergence,

$$
\begin{aligned}
\left(4^{-1} r\right)^{2 d} & \mathbb{E}^{x}\left(\int_{0}^{\infty} \bigotimes_{j=1}^{d} \mathbb{1}_{\left[x_{j}-\pi /(3 r), x_{j}+\pi /(3 r)\right]}\left(X_{s}\right) d s\right) \\
& =\lim _{\alpha \rightarrow 0} \mathbb{E}^{x}\left(\int_{0}^{\infty} e^{-\alpha t}\left(4^{-1} r\right)^{2 d} \bigotimes_{j=1}^{d} \mathbb{1}_{\left[x_{j}-\pi /(3 r), x_{j}+\pi /(3 r)\right]}\left(X_{s}\right) d s\right) \\
& \leqslant \lim _{\alpha \rightarrow 0} \int_{0}^{\infty} e^{-\alpha t} T_{t} g(x) d t \\
& =\lim _{\alpha \rightarrow 0} \int_{0}^{\infty} e^{-\alpha t} d t \int e^{i\langle x, \xi\rangle} \hat{g}(\xi) \lambda_{t}(x, \xi) d \xi \\
& =(8 \pi)^{-d} \lim _{\alpha \rightarrow 0} \int_{0}^{\infty} e^{-\alpha t} \int\left(\bigotimes_{j=1}^{d} \mathbb{1}_{[-r, r]} * \bigotimes_{j=1}^{d} \mathbb{1}_{[-r, r]}\right)(\xi) \lambda_{t}(x, \xi) d \xi d t \\
& =(8 \pi)^{-d} \lim _{\alpha \rightarrow 0} \int_{0}^{\infty} e^{-\alpha t} \int\left(\bigotimes_{j=1}^{d} \mathbb{1}_{[-r, r]} * \bigotimes_{j=1}^{d} \mathbb{1}_{[-r, r]}\right)(\xi) \operatorname{Re} \lambda_{t}(x, \xi) d \xi d t \\
& \leqslant(8 \pi)^{-d} \lim _{\alpha \rightarrow 0} \int_{0}^{\infty} e^{-\alpha t} \int\left(\bigotimes_{j=1}^{d} \mathbb{1}_{[-r, r]} * \bigotimes_{j=1}^{d} \mathbb{1}_{[-r, r]}\right)(\xi)\left|\operatorname{Re} \lambda_{t}(x, \xi)\right| d \xi d t
\end{aligned}
$$

In the penultimate line we have used that the function $\bigotimes_{j=1}^{d} \mathbb{1}_{[-r, r]} * \bigotimes_{j=1}^{d} \mathbb{1}_{[-r, r]}$ is symmetric. Note that for all $\xi \in \mathbb{R}^{d}$,

$$
\left(\bigotimes_{j=1}^{d} \mathbb{1}_{[-r, r]} * \bigotimes_{j=1}^{d} \mathbb{1}_{[-r, r]}\right)(\xi) \leqslant(2 r)^{d}\left(\bigotimes_{j=1}^{d} \mathbb{1}_{[-2 r, 2 r]}\right)(\xi) \leqslant(2 r)^{d} \mathbb{1}_{Q(0,2 r)}(\xi)
$$

This inequality and (2.18) give

$$
\begin{aligned}
\left(\frac{\pi r}{4}\right)^{d} & \mathbb{E}^{x}\left(\int_{0}^{\infty} \bigotimes_{j=1}^{d} \mathbb{1}_{\left[x_{j}-\pi /(3 r), x_{j}+\pi /(3 r)\right]}\left(X_{s}\right) d s\right) \\
& \leqslant \lim _{\alpha \rightarrow 0} \int_{0}^{\infty} e^{-\alpha t} \int_{Q(0,2 r)}\left|\operatorname{Re} \lambda_{t}(x, \xi)\right| d \xi d t \\
& \leqslant \int_{0}^{\infty} \int_{Q(0,2 r)}\left|\operatorname{Re} \lambda_{t}(x, \xi)\right| d \xi d t \\
& \leqslant \int_{0}^{\infty} \int_{\{|\xi| \leqslant 2 r \sqrt{d}\}}\left|\lambda_{t}(x, \xi)\right| d \xi d t \\
& \leqslant \int_{0}^{\infty} \int_{\{|\xi| \leqslant 2 r \sqrt{d}\}} \exp \left(-\frac{t}{16} \inf _{z \in \mathbb{R}^{d}} \operatorname{Re} p(z, 2 \xi)\right) d \xi d t \\
& =16 \int_{\{|\xi| \leqslant 2 r \sqrt{d}\}} \frac{d \xi}{\inf _{z \in \mathbb{R}^{d}} \operatorname{Re} p(z, 2 \xi)} .
\end{aligned}
$$


Therefore, for any $r>0$,

$$
\mathbb{E}^{x}\left(\int_{0}^{\infty} \mathbb{1}_{Q(x, \pi /(3 r))}\left(X_{s}\right) d s\right) \leqslant \frac{4^{d+2}}{(\pi r)^{d}} \int_{\{|\xi| \leqslant 2 r \sqrt{d}\}} \frac{d \xi}{\inf _{z \in \mathbb{R}^{d}} \operatorname{Re} p(z, 2 \xi)} .
$$

Since $r>0$ is arbitrary, the assertion follows because of (1.10).

(iii) Our proof follows Berman's argument; see [5, Chapter V, Theorem 1.1 (1), Page 126] and [4, Section 3]. The occupation measure $\mu_{t}$ of the time interval $[0, t]$, $t>0$, is defined through the relation

$$
\int_{\mathbb{R}^{d}} f(x) \mu_{t}(d x)=\int_{0}^{t} f\left(X_{s}\right) d s \quad \text { for all } f \in B_{b}\left(\mathbb{R}^{d}\right), f \geqslant 0 .
$$

Define the measure $\mu$ by

$$
\mu(d x):=\int_{0}^{\infty} e^{-t} \mu_{t}(d x) d t
$$

in the vague topology of measures. In particular, each $\mu_{t}$ is absolutely continuous with respect to $\mu$ with a density bounded from above by $e^{t}$. We claim that

$$
\int_{\mathbb{R}^{d}} \mathbb{E}^{x}\left(|\widehat{\mu}(\xi)|^{2}\right) d \xi<\infty \quad \text { for every } x \in \mathbb{R}^{d} .
$$

Using Fubini's theorem and then Plancherel's theorem we conclude that, almost surely, $\mu$ has a square-integrable density $\frac{d \mu}{d x}$ with respect to $d x \otimes d \mathbb{P}$. By the definition of $\mu_{t}$ and $\mu$, the local time of the process is just $L(x, t)=e^{t} \times \frac{d \mu}{d x}$ for all $x \in \mathbb{R}^{d}$ and $t \geqslant 0$, and so the required assertion follows.

All that remains to be done is to establish (3.33). From the very definition of $\mu$, one has

$$
\begin{aligned}
& \mathbb{E}^{x}\left(|\widehat{\mu}(\xi)|^{2}\right)=\mathbb{E}^{x}(\widehat{\mu}(\xi) \widehat{\mu}(-\xi)) \\
& =\mathbb{E}^{x}\left[\left(\int_{0}^{\infty} e^{-s} e^{i\left\langle X_{s}, \xi\right\rangle} d s\right)\left(\int_{0}^{\infty} e^{-t} e^{-i\left\langle X_{t}, \xi\right\rangle} d t\right)\right] \\
& =\mathbb{E}^{x}\left(\int_{0}^{\infty} \int_{0}^{\infty} e^{-(s+t)} e^{i\left\langle X_{s}-X_{t}, \xi\right\rangle} d s d t\right) \\
& =\mathbb{E}^{x}\left(\int_{0}^{\infty} \int_{t}^{\infty} e^{-(s+t)} e^{i\left\langle X_{s}-X_{t}, \xi\right\rangle} d s d t\right)+\mathbb{E}^{x}\left(\int_{0}^{\infty} \int_{0}^{t} e^{-(s+t)} e^{i\left\langle X_{s}-X_{t}, \xi\right\rangle} d s d t\right) \\
& =\mathbb{E}^{x}\left(\int_{0}^{\infty} \int_{t}^{\infty} e^{-(s+t)} e^{i\left\langle X_{s}-X_{t}, \xi\right\rangle} d s d t\right)+\mathbb{E}^{x}\left(\int_{0}^{\infty} \int_{s}^{\infty} e^{-(s+t)} e^{i\left\langle X_{s}-X_{t}, \xi\right\rangle} d t d s\right) \\
& =2 \mathbb{E}^{x}\left(\int_{0}^{\infty} \int_{t}^{\infty} e^{-(s+t)} \operatorname{Re} e^{i\left\langle X_{s}-X_{t}, \xi\right\rangle} d s d t\right) \\
& =2 \int_{0}^{\infty} \int_{t}^{\infty} e^{-(s+t)} \operatorname{Re} \mathbb{E}^{x}\left(e^{i\left\langle X_{s}-X_{t}, \xi\right\rangle}\right) d s d t \\
& =2 \int_{0}^{\infty} \int_{t}^{\infty} e^{-(s+t)} \mathbb{E}^{x}\left(\left.\operatorname{Re} \mathbb{E}^{y}\left(e^{i\left\langle X_{s-t}-y, \xi\right\rangle}\right)\right|_{y=X_{t}}\right) d s d t .
\end{aligned}
$$

In the last step we have used the Markov property. From (2.18) we conclude that

$$
\begin{aligned}
\mathbb{E}^{x}\left(|\widehat{\mu}(\xi)|^{2}\right) & \leqslant 2 \int_{0}^{\infty} \int_{t}^{\infty} e^{-(s+t)} \sup _{z \in \mathbb{R}^{d}}\left|\operatorname{Re} \lambda_{s-t}(z, \xi)\right| d s d t \\
& \leqslant 2 \int_{0}^{\infty} \int_{t}^{\infty} e^{-(s+t)} \sup _{z \in \mathbb{R}^{d}}\left|\lambda_{s-t}(z, \xi)\right| d s d t
\end{aligned}
$$




$$
\begin{aligned}
& \leqslant 2 \int_{0}^{\infty} \int_{t}^{\infty} e^{-(s+t)-\frac{1}{16}(s-t) \inf _{z \in \mathbb{R}^{d}} \operatorname{Re} p(z, \xi)} d s d t \\
& =2 \int_{0}^{\infty} \int_{t}^{\infty} e^{-(s-t)-\frac{1}{16}(s-t) \inf _{z \in \mathbb{R}^{d}} \operatorname{Re} p(z, \xi)} d s e^{-2 t} d t \\
& =\frac{16}{16+\inf _{z \in \mathbb{R}^{d}} \operatorname{Re} p(z, \xi)} .
\end{aligned}
$$

This estimate and the assumption (1.11) show that (3.33) holds.

Remark 3.1. (i) A close inspection of the proofs of Theorem 1.2 (ii) and (iii) shows that the transience and the existence of local times for a Feller process only depend on $\operatorname{Re} \lambda_{t}(x, \xi)$, i.e. the real part of the characteristic function. This is familiar from the theory of Lévy processes.

(ii) If for every $x \in \mathbb{R}^{d}$ the symbol $\operatorname{Re} p(x, \xi)$ is a function of $|\xi|$ which is unbounded in $\xi$, i.e. if for every $x \in \mathbb{R}^{d}, \operatorname{Re} p(x, \xi)=\operatorname{Re} p(x,|\xi|)$ and

$$
\varliminf_{|\xi| \rightarrow \infty} \operatorname{Re} p(x,|\xi|)=\infty
$$

then we can replace the condition 'for every $r>0$ ' in (1.10) by 'for some $r>0$ '.

This can be seen from the following argument: first,

$$
\int_{\{|\xi| \leqslant r\}} \frac{d \xi}{\inf _{z \in \mathbb{R}^{d}} \operatorname{Re} p(z, \xi)}=\infty
$$

is equivalent to saying that

$$
\int_{\{|\xi| \leqslant r\}} \frac{d \xi}{\sup _{z \in \mathbb{R}^{d}}(-\operatorname{Re} p(z, \xi))}=-\infty .
$$

Now, if there exists $r_{0}>0$ such that

$$
\int_{\left\{|\xi| \leqslant r_{0}\right\}} \frac{d \xi}{\inf _{z \in \mathbb{R}^{d}} \operatorname{Re} p(z, \xi)}<\infty
$$

and

$$
\int_{\{|\xi| \leqslant r\}} \frac{d \xi}{\inf _{z \in \mathbb{R}^{d}} \operatorname{Re} p(z, \xi)}=\infty \quad \text { for all } r>r_{0},
$$

then,

and

$$
\int_{\left\{|\xi| \leqslant r_{0}\right\}} \frac{d \xi}{\sup _{z \in \mathbb{R}^{d}}(-\operatorname{Re} p(z, \xi))}<-\infty
$$

$$
\int_{\{|\xi| \leqslant r\}} \frac{d \xi}{\sup _{z \in \mathbb{R}^{d}}(-\operatorname{Re} p(z, \xi))}=-\infty \quad \text { for all } r>r_{0} .
$$

Hence,

$$
\int_{\left\{r_{0} \leqslant|\xi| \leqslant r\right\}} \frac{d \xi}{\sup _{z \in \mathbb{R}^{d}}(-\operatorname{Re} p(z, \xi))}=-\infty \text { for all } r>r_{0} .
$$

Thus, there exists a sequence $\left(\xi_{n}\right)_{n \geqslant 1} \subset\left\{z \in \mathbb{R}^{d}: r_{0} \leqslant|z| \leqslant r\right\}$ such that

$$
\lim _{n \rightarrow \infty} \sup _{z \in \mathbb{R}^{d}} \operatorname{Re} p\left(z, \xi_{n}\right)=0 \text {. }
$$

In particular, for all $x \in \mathbb{R}^{d}, \lim _{n \rightarrow \infty} \operatorname{Re} p\left(x, \xi_{n}\right)=0$.

By compactness, there is a subsequence $\left(\xi_{n}^{\prime}\right)_{n \geqslant 1}$ of $\left(\xi_{n}\right)_{n \geqslant 1}$ such that $\lim _{n \rightarrow \infty} \xi_{n}^{\prime}$ $=\xi_{0}$. Since the function $\xi \mapsto \operatorname{Re} p(x, \xi)$ is continuous for any fixed $x \in \mathbb{R}^{d}$, we get that $\operatorname{Re} p\left(x, \xi_{0}\right)=0$ for every $x \in \mathbb{R}^{d}$. Thus, for any $x \in \mathbb{R}^{d}$ and $\eta \in \mathbb{R}^{d}$ 
with $|\eta|=\left|\xi_{0}\right|, \operatorname{Re} p(x, \eta)=0$. Since $\sqrt{\operatorname{Re} p(x, \cdot)}$ is subadditive, $\xi \mapsto \operatorname{Re} p(x, \xi)$ is periodic. Because of (1.6), there is a constant $C=C\left(\xi_{0}\right)$ such that

$$
\sup _{x, \xi \in \mathbb{R}^{d}} \operatorname{Re} p(x, \xi) \leqslant C,
$$

which cannot be the case since $\operatorname{Re} p(x, \xi)$ is unbounded.

\subsection{Examples.}

Feller processes with real symbol obtained by variable order subordination. Let $\psi$ be a real-valued negative definite function on $\mathbb{R}^{d}$ such that $\psi(0)=0$. Let $f: \mathbb{R}^{d} \times[0, \infty) \rightarrow[0, \infty)$ be a measurable function such that $\sup _{x} f(x, s) \leqslant$ $c(1+s)$ for some constant $c>0$, and for fixed $x \in \mathbb{R}^{d}$ the function $s \mapsto f(x, s)$ is a Bernstein function with $f(x, 0)=0$. Bernstein functions are the characteristic Laplace exponents of subordinators; our standard reference is the monograph 33. Then,

$$
q(x, \xi):=f(x, \psi(\xi))
$$

is a real-valued symbol satisfying (1.6). Since $f(x, s)=s^{r(x)}$, where $r: \mathbb{R}^{d} \rightarrow[0,1]$ is a possible choice for $f$, this class includes symbols describing variable (fractional) order of differentiation or variable order fractional powers. We refer to [11] and the references therein for more details on Feller semigroups obtained by variable order subordination. According to Theorem 1.2 and Remark 3.1, we see

Corollary 3.2. Let $\left(X_{t}\right)_{t \geqslant 0}$ be a Feller process with the symbol $q(x, \xi)=f(x, \psi(\xi))$ above. Set $f_{0}(s):=\inf _{x \in \mathbb{R}^{d}} f(x, s)$ for $s \in[0, \infty)$. Then, we have

(i) If

$$
\lim _{|\xi| \rightarrow \infty} \frac{f_{0}(\psi(\xi))}{\log (1+|\xi|)}=\infty
$$

then the corresponding Feller semigroup $\left(T_{t}\right)_{t \geqslant 0}$ is ultracontractive and has the strong Feller property.

(ii) If

$$
\int_{\{|\xi| \leqslant r\}} \frac{d \xi}{f_{0}(\psi(\xi))}<\infty \quad \text { for every } r>0
$$

then the Feller process $\left(X_{t}\right)_{t \geqslant 0}$ is transient.

(iii) If

$$
\int \frac{d \xi}{1+f_{0}(\psi(\xi))}<\infty
$$

then the Feller process $\left(X_{t}\right)_{t \geqslant 0}$ has local times.

If the symbol $\psi(\xi)$ only depends on $|\xi|$, i.e. if $\psi(\xi)=\phi(|\xi|)$ for some function $\phi$, then it is enough to assume that the condition in (ii) holds for some $r>0$.

Rich Bass' stable-like processes. A stable-like process on $\mathbb{R}^{d}$ is a Feller process, whose generator has the same form as that of a rotationally symmetric stable Lévy motion, but the index of 'stability' depends on the state space; see [1]. The infinitesimal generator is of the form

$$
L^{(\alpha)} u(x)=\int_{z \neq 0}\left(u(x+z)-u(x)-\langle\nabla u(x), z\rangle \mathbb{1}_{\{|z| \leqslant 1\}}\right) \frac{C_{\alpha(x)}}{|z|^{d+\alpha(x)}} d z, \quad u \in C_{b}^{2}\left(\mathbb{R}^{d}\right),
$$


where $0<\alpha(x)<2$ and $C_{\alpha(x)}$ is a constant defined through the Lévy-Khintchine formula

$$
|\xi|^{\alpha(x)}=C_{\alpha(x)} \int_{z \neq 0}(1-\cos \langle\xi, z\rangle) \frac{d z}{|z|^{d+\alpha(x)}}
$$

i.e.

$$
C_{\alpha(x)}=\alpha(x) 2^{\alpha(x)-1} \Gamma((\alpha(x)+d) / 2) /\left(\pi^{d / 2} \Gamma(1-\alpha(x) / 2)\right) ;
$$

see [3, Exercise 18.23, Page 184]. In other words, the operator $L^{(\alpha)}$ can be regarded as a pseudodifferential operator of variable order with symbol $|\xi|^{\alpha(x)}$, i.e. $L^{(\alpha)}=$ $-(-\Delta)^{\alpha(x) / 2}$.

Theorem 3.3. Assume that $\alpha(x) \in C_{b}^{1}\left(\mathbb{R}^{d}\right)$ such that $0<\underline{\alpha}=\inf \alpha \leqslant \alpha(x) \leqslant$ $\sup \alpha=\bar{\alpha}<2$. Then, there exists a Feller process $\left(X_{t}\right)_{t \geqslant 0}$ (which we call a stablelike process in the sense of $R$. Bass) having the symbol $|\xi|^{\alpha(x)}$, such that the following statements hold.

(i) The Feller semigroup $\left(T_{t}\right)_{t \geqslant 0}$ of $\left(X_{t}\right)_{t \geqslant 0}$ has the strong Feller property, and the transition probability $P(t, x, d y)$ of $\left(X_{t}\right)_{t \geqslant 0}$ has a density function $p(t, x, y)$ with respect to Lebesgue measure; moreover

$$
\sup _{x, y \in \mathbb{R}^{d}} p(t, x, y) \leqslant \begin{cases}C t^{-d / \underline{\alpha}} & \text { for small } t \ll 1 ; \\ C t^{-d / \bar{\alpha}} & \text { for large } t \gg 1 .\end{cases}
$$

(ii) If $d \geqslant 2$, then the process $\left(X_{t}\right)_{t \geqslant 0}$ is transient.

(iii) If $d=1$ and $\sup _{|x| \geqslant K} \alpha(x) \in(0,1)$ for some constant $K>0$, then the process $\left(X_{t}\right)_{t \geqslant 0}$ is transient.

(iv) If $d=1$ and $\inf _{x \in \mathbb{R}} \alpha(x) \in(1,2)$, the process $\left(X_{t}\right)_{t \geqslant 0}$ has local times.

Before we begin with the proof of Theorem 3.3, a few words on related work on stable-like processes are appropriate.

Remark 3.4. (i) Under the condition that $\alpha(\cdot) \in C_{b}^{\infty}\left(\mathbb{R}^{d}\right)$, the strong Feller property of stable-like processes has been established in [34, Theorem 3.3]. In addition to this, our result provides an upper bound for on-diagonal estimates of the heat kernel of stable-like processes. Note that a stable-like process is not symmetric; i.e., Dirichlet form methods fail if we want to derive estimates as in Theorem 3.3 (i).

(ii) If $\alpha(x)$ is Dini continuous and $\inf _{x \in \mathbb{R}} \alpha(x) \in(1,2)$, the existence of local times for stable-like processes was shown by Bass [2, Theorem 2.1]. Bass' technique is different from ours.

(iii) Recurrence and transience of a particular class of one-dimensional stablelike processes (with discontinuous exponents) have been studied in [6] using an overshoot approach under the assumption that the underlying process is a Lebesgueirreducible T-process. Although the setting in [6, Corollary 5.5] is different from the situation here, we remark that our proof shows that a stable-like process with $\alpha(x) \in C_{b}^{1}\left(\mathbb{R}^{d}\right)$ and $0<\underline{\alpha}=\inf \alpha \leqslant \alpha(x) \leqslant \sup \alpha=\bar{\alpha}<2$ is a Lebesgue-irreducible T-process.

Proof of Theorem 3.3. According to [1, Corollary 2.3] the solution to the martingale problem for $\left(L^{(\alpha)}, C_{0}^{\infty}\left(\mathbb{R}^{d}\right)\right)$ is well posed. Therefore there exists a unique strong Markov process $\left(\left(X_{t}\right)_{t \geqslant 0},\left(\mathbb{P}^{x}\right)_{x \in \mathbb{R}^{d}}\right)$ for which $\mathbb{P}^{x}$ solves the martingale problem for 
$\left(L^{(\alpha)}, C_{0}^{\infty}\left(\mathbb{R}^{d}\right)\right)$ at each point $x \in \mathbb{R}^{d}$. For any $t \geqslant 0, x \in \mathbb{R}^{d}$ and $f \in B_{b}\left(\mathbb{R}^{d}\right)$, we define

$$
T_{t} f(x)=\mathbb{E}^{x}\left(f\left(X_{t}\right)\right) .
$$

From [1, Propositions 6.1 and 6.2], we know that $\left(T_{t}\right)_{t \geqslant 0}$ is a Markov semigroup which has the $C_{b}$-Feller property; that is, for any $t \geqslant 0, T_{t}$ maps the set of bounded continuous functions into itself. By Proposition 4.4 we see that $T_{t}$ enjoys the Feller property; i.e., $T_{t}$ maps the set of continuous functions vanishing at infinity into itself. Note that the uniqueness of the solution for the martingale problem indicates that $C_{c}^{\infty}\left(\mathbb{R}^{d}\right)$ is contained in the extended domain of the operator $L^{(\alpha)}$. On the other hand, it is easy to check that, under our assumptions on the index function $\alpha$, we have $L^{(\alpha)} u \in C_{\infty}\left(\mathbb{R}^{d}\right)$ for any $u \in C_{c}^{\infty}\left(\mathbb{R}^{d}\right)$. Thus, Proposition 4.1 shows that $C_{c}^{\infty}\left(\mathbb{R}^{d}\right)$ actually is contained in the domain of the operator $L^{(\alpha)}$. Therefore, (i), (ii) and (iv) follow from Theorem 1.2 ,

To prove the assertion (iii) we need a few auxiliary results on stable-like processes. Let $P(t, x, d y)$ be the transition function of $\left(X_{t}\right)_{t \geqslant 0}$ and denote its density by $p(t, x, y)$. Note that $x \mapsto C_{\alpha(x)}$ is a positive function of class $C_{b}^{\infty}\left(\mathbb{R}^{d}\right)$. From [22, Theorem 5.1 and its Corollary, Pages 759-760] we know that $p(t, x, y)$ is strictly positive everywhere on $(0, \infty) \times \mathbb{R}^{d} \times \mathbb{R}^{d}$. Therefore, $\left(X_{t}\right)_{t \geqslant 0}$ is Lebesgue irreducible; i.e., for any Borel measurable set $A$ with $\operatorname{Leb}(A)>0$ and $x \in \mathbb{R}^{d}$, $\mathbb{E}^{x}\left(\int_{0}^{\infty} \mathbb{1}_{A}\left(X_{t}\right) d t\right)>0$. Recall that a Markov process $\left(X_{t}\right)_{t \geqslant 0}$ is Harris recurrent if for any Borel measurable set $A$ with $\operatorname{Leb}(A)>0$ and $x \in \mathbb{R}^{d}, \mathbb{E}^{x}\left(\int_{0}^{\infty} \mathbb{1}_{A}\left(X_{t}\right) d t\right)=$ $\infty$. The Lebesgue irreducibility and the strong Feller property yield that the stablelike process $\left(X_{t}\right)_{t \geqslant 0}$ is either Harris recurrent or transient; see e.g. [24, Theorem 3.2 (a)] and [36, Theorem 2.3]. Moreover, we know from [23, Theorem 3.3] that $\left(X_{t}\right)_{t \geqslant 0}$

is Harris recurrent if, and only if, $\mathbb{P}^{x}\left(\sigma_{\overline{B(0, R)}}<\infty\right)=1$ for every $x \in \mathbb{R}^{d}$; is transient if, and only if, $\mathbb{P}^{x}\left(\sigma_{\overline{B(0, R)}}<\infty\right)<1$ for some $x \in \mathbb{R}^{d}$,

where $\sigma_{\overline{B(0, R)}}$ is the first entrance time of the process into $\overline{B(0, R)}$ and $R>0$ is any fixed radius. From these characterizations, we conclude that any two stablelike processes which coincide outside some compact set have the same (Harris) recurrence and transience behaviour; see [6, Theorems 4.6 and 4.7].

Now we can use Theorem 1.2 (ii) to infer that a one-dimensional stable-like process is transient if $\sup _{x \in \mathbb{R}} \alpha(x) \in(0,1)$. Therefore, (iii) follows from this conclusion and the remark above.

\section{Appendix}

Let $\left(X_{t}\right)_{t \geqslant 0}$ be a Feller process with generator $(A, D(A))$ and semigroup $\left(T_{t}\right)_{t \geqslant 0}$. Let us first comment on the assumption that

$$
\text { the test functions } C_{c}^{\infty}\left(\mathbb{R}^{d}\right) \text { are contained in the domain } D(A)
$$

of the Feller generator $A$. Usually (4.34) is not easy to verify in applications; on the other hand, we do not know many nontrivial examples of Feller processes which do not satisfy (4.34). In what follows, we will make full use of the extended domain of the Feller generator $A$, which is easier to deal with than the domain $D(A)$. 
Recall that for a strong Markov process $\left(X_{t}\right)_{t \geqslant 0}$ on $\mathbb{R}^{d}$ with infinitesimal generator $(A, D(A))$, the extended domain $\widetilde{D}(A)$ is defined by

$$
\begin{aligned}
& \widetilde{D}(A)=\left\{u \in B\left(\mathbb{R}^{d}\right): \text { there is a measurable function } g\right. \text { such that } \\
& \left.\left(u\left(X_{t}\right)-\int_{0}^{t} g\left(X_{s}\right) d s, \mathcal{F}_{t}\right)_{t \geqslant 0} \text { is a local martingale under } \mathbb{P}^{x}\right\},
\end{aligned}
$$

where $\mathcal{F}_{t}:=\sigma\left(X_{s}: s \leqslant t\right)$ is the natural filtration of the process $\left(X_{t}\right)_{t \geqslant 0}$, and $B\left(\mathbb{R}^{d}\right)$ is the space of Borel measurable functions on $\mathbb{R}^{d}$. The function $g$ appearing in the definition of $\widetilde{D}(A)$ need not be unique; cf. [10, Chapter 1, Page 24]. If, however, $A u$ can be defined, $g=A u$ is admissible; in particular, $D(A) \subset \widetilde{D}(A)$. Conversely, we can use the situation where $g$ is unique to extend the operator $(A, D(A))$. The concept of extended domain is similar to the full generator for a contraction semigroup in [10, Chapter 1, Pages 23-24].

For a Feller generator $(A, D(A))$ such that $C_{c}^{\infty}\left(\mathbb{R}^{d}\right) \subset D(A)$ one has $C_{c}^{\infty}\left(\mathbb{R}^{d}\right) \subset$ $\widetilde{D}(A)$; see [10, Chapter 4, Proposition 1.7] and [31, Lemma 2.3 and Corollary 3.6]. On the other hand, the condition $C_{c}^{\infty}\left(\mathbb{R}^{d}\right) \subset D(A)$ along with the assumption (1.6) implies that $C_{c}^{\infty}\left(\mathbb{R}^{d}\right) \subset C_{b}^{2}\left(\mathbb{R}^{d}\right) \subset \widetilde{D}(A)$; see Proposition 4.2 below for the simple proof of the assertion that $C_{b}^{\infty}\left(\mathbb{R}^{d}\right) \subset \widetilde{D}(A)$, where $C_{b}^{\infty}\left(\mathbb{R}^{d}\right)$ is the space of arbitrarily often differentiable functions such that the function and its derivatives are bounded. Conversely, we have

Proposition 4.1. Let $\left(X_{t}\right)_{t \geqslant 0}$ be a Feller process with generator $(A, D(A))$. Suppose that $C_{c}^{\infty}\left(\mathbb{R}^{d}\right) \subset \widetilde{D}(A)$, and that for any $u \in C_{c}^{\infty}\left(\mathbb{R}^{d}\right)$ there is (an extension of A) such that $A u$ is well defined and in $C_{\infty}\left(\mathbb{R}^{d}\right)$, the space of continuous functions vanishing at infinity. If the process $\left(X_{t}\right)_{t \geqslant 0}$ is conservative, then $C_{c}^{\infty}\left(\mathbb{R}^{d}\right) \subset D(A)$.

Proof. The Feller semigroup $\left(T_{t}\right)_{t \geqslant 0}$ has a unique extension on $B_{b}\left(\mathbb{R}^{d}\right)$ (the space of the bounded Borel measurable functions); cf. [30, Section 3]. For simplicity, we still denote by $\left(T_{t}\right)_{t \geqslant 0}$ this extension. Since the process $\left(X_{t}\right)_{t \geqslant 0}$ is conservative, $T_{t} 1=1$ for every $t \geqslant 0$. According to [30, Corollary 3.4], $t \mapsto T_{t} u$ is for all $u \in C_{b}\left(\mathbb{R}^{d}\right)$ continuous with respect to locally uniform convergence.

Let $\tau_{B(x, r)}$ be the first exit time of the process from the open ball $B(x, r)$. Since $C_{c}^{\infty}\left(\mathbb{R}^{d}\right) \subset \widetilde{D}(A)$, for any $x \in \mathbb{R}^{d}, r>0$ and $u \in C_{c}^{\infty}\left(\mathbb{R}^{d}\right)$,

$$
\mathbb{E}^{x}\left(u\left(X_{t \wedge \tau_{B(x, r)}}\right)-\int_{0}^{t \wedge \tau_{B(x, r)}} A u\left(X_{s}\right) d s\right)=u(x) .
$$

Since $\left(X_{t}\right)_{t \geqslant 0}$ is conservative, $\tau_{B(x, r)} \stackrel{r \rightarrow \infty}{\longrightarrow} \infty$. Thus, we can use the dominated convergence theorem to find that for all $u \in C_{c}^{\infty}\left(\mathbb{R}^{d}\right)$,

$$
\mathbb{E}^{x}\left(u\left(X_{t}\right)-\int_{0}^{t} A u\left(X_{s}\right) d s\right)=u(x) .
$$

Pick $x \in \mathbb{R}^{d}$; by the continuity of $t \mapsto T_{t}(A u)(x)$,

$$
\lim _{t \rightarrow 0} \frac{\mathbb{E}^{x}\left(u\left(X_{t}\right)-u(x)\right)}{t}=\lim _{t \rightarrow 0} \frac{1}{t}\left(\int_{0}^{t} T_{s}(A u)(x) d s\right)=A u(x) .
$$


Thus, $u$ belongs to the domain of the weak infinitesimal generator of the process $X_{t}$. The required assertion follows from [27, Lemma 31.7, Page 209].

Next, we present a consequence of the assumption $C_{c}^{\infty}\left(\mathbb{R}^{d}\right) \subset D(A)$ for Feller processes.

Proposition 4.2. Let $\left(X_{t}\right)_{t \geqslant 0}$ be a Feller process with generator $(A, D(A))$ and semigroup $\left(T_{t}\right)_{t \geqslant 0}$. Assume that $C_{c}^{\infty}\left(\mathbb{R}^{d}\right) \subset D(A)$ so that $\left.A\right|_{C_{c}^{\infty}\left(\mathbb{R}^{d}\right)}$ is a pseudodifferential operator $-p(\cdot, D)$ with symbol $p(x, \xi)$. If (1.6) is satisfied, then

$$
T_{t} e_{\xi}(x)=e_{\xi}(x)+\int_{0}^{t} T_{s} A e_{\xi}(x) d s
$$

holds for all $t>0$ and $x, \xi \in \mathbb{R}^{d}$, where $e_{\xi}(x)=e^{i\langle\xi, x\rangle}$.

Proof. Denote by $C_{b}^{\infty}\left(\mathbb{R}^{d}\right)$ the space of arbitrarily often differentiable functions such that the function and its derivatives are bounded. First we prove that $C_{b}^{\infty}\left(\mathbb{R}^{d}\right)$ is contained in the extended domain $\widetilde{D}(A)$ of the Feller generator $A$.

Let $(b(x), a(x), \nu(x, d z))_{x \in \mathbb{R}^{d}}$ be the Lévy characteristics of the symbol $p(x, \xi)$ given by (1.5); under the assumption (1.6),$c(x) \equiv 0$. Then $A$ has the following representation as an integro-differential operator:

$$
\begin{aligned}
L f(x)=\frac{1}{2} & \sum_{j, k=1}^{d} a_{j k}(x) \partial_{j k} f(x)+\sum_{j=1}^{d} b_{j}(x) \partial_{j} f(x) \\
& +\int_{z \neq 0}\left(f(x+z)-f(x)-\langle\nabla f(x), z\rangle \mathbb{1}_{\{|z| \leqslant 1\}}\right) \nu(x, d z) .
\end{aligned}
$$

For all $u \in C_{c}^{\infty}\left(\mathbb{R}^{d}\right)$ we have $-p(x, D) u(x)=L u(x), x \in \mathbb{R}^{d}$, cf. [31, (2.7) and Corollary 2.4], and by [31, Lemma 2.3 and Corollary 3.6] we have $C_{b}^{2}\left(\mathbb{R}^{d}\right) \subset \widetilde{D}(A)$. On the other hand, [31, Lemma 2.3 and Corollary 3.6] also show that $(L, D(L))$ is the unique extension of the Feller generator $A$ onto $C_{b}^{2}\left(\mathbb{R}^{d}\right)$ such that $\|L u\|_{\infty} \leqslant C\|u\|_{C_{b}^{2}}$ holds for all $u \in C_{b}^{2}\left(\mathbb{R}^{d}\right)$ and some constant $C>0$; here $\|u\|_{C_{b}^{2}}:=\sum_{|\alpha| \leqslant 2}\left\|\partial^{\alpha} u\right\|_{\infty}$.

Let $\chi \in C_{c}^{\infty}\left(\mathbb{R}^{d}\right)$ be a smooth cutoff function such that $\mathbb{1}_{B(0,1)}(y) \leqslant \chi(y) \leqslant$ $\mathbb{1}_{B(0,2)}(y)$ for $y \in \mathbb{R}^{d}$. For $u \in C_{b}^{\infty}\left(\mathbb{R}^{d}\right)$ we define $u_{n}^{x}(y):=\chi((y-x) / n) u(y)$. Then, $u_{n}^{x} \in C_{c}^{\infty}\left(\mathbb{R}^{d}\right)$ for every $n \geqslant 1$. By the Taylor formula and the Leibniz rule we see that for any compact set $K \subset \mathbb{R}^{d}$ there exists a positive constant $C:=C(K, u, n)$ such that $\left|L u_{n}^{x}(y)\right| \leqslant C$ for all $y \in K$. Let $\tau_{B(x, r)}$ be the first exit time of the process from the open ball $B(x, r)$. By the bounded convergence theorem and the fact that $C_{c}^{\infty}\left(\mathbb{R}^{d}\right) \subset \widetilde{D}(L)$, we find for all $x \in \mathbb{R}^{d}$ and $r, t>0$,

$$
\begin{aligned}
\mathbb{E}^{x}\left(u\left(X_{t \wedge \tau_{B(x, r)}}\right)-u(x)\right) & =\lim _{n \rightarrow \infty} \mathbb{E}^{x}\left(u_{n}^{x}\left(X_{t \wedge \tau_{B(x, r)}}\right)-u_{n}^{x}(x)\right) \\
& =\lim _{n \rightarrow \infty} \mathbb{E}^{x}\left(\int_{0}^{t \wedge \tau_{B(x, r)}} L u_{n}^{x}\left(X_{s}\right) d s\right) \\
& =\lim _{n \rightarrow \infty} \mathbb{E}^{x}\left(\int_{\left(0, t \wedge \tau_{B(x, r)}\right)} L u_{n}^{x}\left(X_{s}\right) d s\right) .
\end{aligned}
$$


By the dominated convergence theorem, we may interchange limit and integration to get

$$
\begin{aligned}
\mathbb{E}^{x}\left(u\left(X_{t \wedge \tau_{B(x, r)}}\right)-u(x)\right) & =\mathbb{E}^{x}\left(\int_{\left(0, t \wedge \tau_{B(x, r)}\right)} \lim _{n \rightarrow \infty} L u_{n}^{x}\left(X_{s}\right) d s\right) \\
& =\mathbb{E}^{x}\left(\int_{\left(0, t \wedge \tau_{B(x, r)}\right)} L u\left(X_{s}\right) d s\right) .
\end{aligned}
$$

Therefore, for any $x \in \mathbb{R}^{d}$ and $r>0$,

$$
\mathbb{E}^{x}\left(u\left(X_{t \wedge \tau_{B(x, r)}}\right)-\int_{0}^{t \wedge \tau_{B(x, r)}} L u\left(X_{s}\right) d s\right)=u(x) .
$$

Because of (1.6), the process $\left(X_{t}\right)_{t \geqslant 0}$ is conservative. Therefore, $\tau_{B(x, r)} \stackrel{r \rightarrow \infty}{\longrightarrow} \infty$, and we find by dominated convergence for all $u \in C_{b}^{\infty}\left(\mathbb{R}^{d}\right)$ that

$$
\mathbb{E}^{x}\left(u\left(X_{t}\right)-\int_{0}^{t} L u\left(X_{s}\right) d s\right)=u(x) .
$$

Note that $(L, D(L))$ is the unique extension of $(A, D(A)), C_{b}^{\infty}\left(\mathbb{R}^{d}\right) \subset D(L)$. Now the Markov property shows that $u \in \widetilde{D}(A)$.

If we set $u(x)=e_{\xi}(x)$ and use that $L e_{\xi}(x)=A e_{\xi}(x)$, the assertion follows.

If $C_{c}^{\infty}\left(\mathbb{R}^{d}\right) \subset D(A)$, the following result can be deduced from 31, Lemmas 4.1 and Lemma 5.1].

Proposition 4.3. Let $\left(X_{t}\right)_{t \geqslant 0}$ be a Feller process with generator $(A, D(A))$ such that $C_{c}^{\infty}\left(\mathbb{R}^{d}\right) \subset D(A)$ and (1.6) holds. Let $\tau_{B(x, r)}$ be the first exit time of the process from the open ball $B(x, r)$. Then, for any $x \in \mathbb{R}^{d}$ and $r, t>0$,

$$
\begin{aligned}
\mathbb{P}^{x}\left(\tau_{B(x, r)} \leqslant t\right) & \leqslant c t \sup _{|y-x| \leqslant r} \sup _{|\xi| \leqslant 1 / r}|p(y, \xi)| \\
& \leqslant c t \sup _{|\xi| \leqslant 1 / r} \sup _{z \in \mathbb{R}^{d}}|p(z, \xi)|
\end{aligned}
$$

with an absolute constant $c>0$.

Let $\left(X_{t}\right)_{t \geqslant 0}$ be a strong Markov process with semigroup $\left(T_{t}\right)_{t \geqslant 0}$ and generator $A$. Assume that the semigroup $\left(T_{t}\right)_{t \geqslant 0}$ has the $C_{b}$-Feller property, i.e. $T_{t}\left(C_{b}\left(\mathbb{R}^{d}\right)\right) \subset$ $C_{b}\left(\mathbb{R}^{d}\right)$ for all $t>0$, where $C_{b}\left(\mathbb{R}^{d}\right)$ is the set of bounded and continuous functions on $\mathbb{R}^{d}$. Moreover, we assume that $C_{c}^{\infty}\left(\mathbb{R}^{d}\right)$ is contained in the extended domain $\widetilde{D}(A)$ of the operator $A$ and that $\left.A\right|_{C_{c}^{\infty}\left(\mathbb{R}^{d}\right)}=-p(\cdot, D)$, where $-p(\cdot, D)$ is a pseudodifferential operator with symbol $p(x, \xi)$. Then, for any $u \in C_{c}^{\infty}\left(\mathbb{R}^{d}\right)$,

$$
\left(u\left(X_{t}\right)-\int_{0}^{t}\left(-p\left(X_{s}, D\right) u\left(X_{s}\right)\right) d s, \mathcal{F}_{t}\right)_{t \geqslant 0} \text { is a local martingale under } \mathbb{P}^{x} \text {. }
$$

Furthermore, we have the following simple condition on the symbol $p(x, \xi)$ to yield the $\left(C_{\infty^{-}}\right)$Feller property of $\left(T_{t}\right)_{t \geqslant 0}$.

Proposition 4.4. If the symbol $p(x, \xi)$ satisfies

$$
\varlimsup_{r \rightarrow \infty} \sup _{|x| \leqslant r} \sup _{|\xi| \leqslant 1 / r}|p(x, \xi)|=0,
$$

then $\left(T_{t}\right)_{t \geqslant 0}$ has the Feller property; i.e., $T_{t}\left(C_{\infty}\left(\mathbb{R}^{d}\right)\right) \subset C_{\infty}\left(\mathbb{R}^{d}\right)$ for every $t \geqslant 0$, where $C_{\infty}\left(\mathbb{R}^{d}\right)$ is the set of continuous functions on $\mathbb{R}^{d}$ vanishing at infinity. 
Proof. Since $\sqrt{|p(x, \cdot)|}$ is, for any fixed $x \in \mathbb{R}^{d}$, subadditive, it is not hard to see that (4.37) is equivalent to

$$
\varlimsup_{r \rightarrow \infty} \sup _{|x| \leqslant \gamma r} \sup _{|\xi| \leqslant 1 / r}|p(x, \xi)|=0, \quad \gamma \geqslant 1 .
$$

A close inspection of the proof of Proposition 4.3 shows that (4.36a) also holds in the present setting. For every $f \in C_{\infty}\left(\mathbb{R}^{d}\right)$ we see by the $C_{b}$-Feller property that $T_{t} f \in C_{b}\left(\mathbb{R}^{d}\right)$ is continuous. We have to study the behaviour of $T_{t} f(x)$ as $|x| \rightarrow \infty$. If $f \in C_{\infty}\left(\mathbb{R}^{d}\right)$, we find for every $\varepsilon>0$ some $r_{1}:=r_{1}(\varepsilon, f)>0$ such that

$$
|f(y)| \leqslant \varepsilon / 2 \text { for all }|y| \geqslant r_{1} .
$$

Because of (4.38), there is some constant $r_{2}:=r_{2}(\varepsilon, f)>r_{1}>0$ such that

$$
\sup _{|z| \leqslant 3|y| / 2} \sup _{|\xi| \leqslant 2 /|y|}|p(z, \xi)| \leqslant \frac{\varepsilon}{2 c t\left(\|f\|_{\infty}+1\right)} \quad \text { for all }|y| \geqslant r_{2}
$$

( $c$ is the constant appearing in Proposition 4.3). By 4.36a) we find for $y \in \mathbb{R}^{d}$ with $|y| \geqslant 2 r_{2}$

$$
\begin{aligned}
\left|\left(T_{t} f\right)(y)\right| & \leqslant \int|f(z)| \mathbb{P}^{y}\left(X_{t} \in d z\right) \\
& =\int_{B\left(0, r_{2}\right)}|f(z)| \mathbb{P}^{y}\left(X_{t} \in d z\right)+\int_{B^{c}\left(0, r_{2}\right)}|f(z)| \mathbb{P}^{y}\left(X_{t} \in d z\right) \\
& \leqslant\|f\|_{\infty} \mathbb{P}^{y}\left(\left|X_{t}\right| \leqslant r_{2}\right)+\varepsilon / 2 \\
& \leqslant\|f\|_{\infty} \mathbb{P}^{y}\left(\left|X_{t}-y\right| \geqslant|y|-r_{2}\right)+\varepsilon / 2 \\
& \leqslant\|f\|_{\infty} \mathbb{P}^{y}\left(\sup _{s \leqslant t}\left|X_{s}-y\right| \geqslant|y| / 2\right)+\varepsilon / 2 \\
& \leqslant c t\|f\|_{\infty} \sup _{|z-y| \leqslant|y| / 2} \sup _{|\xi| \leqslant 2 /|y|}|p(z, \xi)|+\varepsilon / 2 \\
& \leqslant c t\|f\|_{\infty} \sup _{|z| \leqslant 3|y| / 2} \sup _{|\xi| \leqslant 2 /|y|}|p(z, \xi)|+\varepsilon / 2 \\
& \leqslant \varepsilon,
\end{aligned}
$$

which shows that $\lim _{|y| \rightarrow \infty} T_{t} f(y)=0$ for all $f \in C_{\infty}\left(\mathbb{R}^{d}\right)$.

The following statement presents a general connection between a $C_{b}$-Feller and a $\left(C_{\infty^{-}}\right)$Feller semigroup.

Proposition 4.5. (i) Suppose that $\left(T_{t}\right)_{t \geqslant 0}$ is a Feller semigroup. If $T_{t} 1 \in C_{b}\left(\mathbb{R}^{d}\right)$ for every fixed $t \geqslant 0$, then $\left(T_{t}\right)_{t \geqslant 0}$ is a $C_{b}$-Feller semigroup. In particular, any conservative Feller semigroup, i.e. for $t \geqslant 0, T_{t} 1=1$, is a $C_{b}$-Feller semigroup.

(ii) Let $\left(T_{t}\right)_{t \geqslant 0}$ be a $C_{b}$-Feller semigroup and $(P(t, x, d y))_{t>0}$ the corresponding family of kernels; i.e., for any $t>0, x \in \mathbb{R}^{d}$ and $u \in C_{b}\left(\mathbb{R}^{d}\right), T_{t} u(x)=\int u(y) P(t, x, d y)$. Then, $\left(T_{t}\right)_{t>0}$ is a Feller semigroup if, and only if, for all $t>0$ and all bounded sets $B \in \mathcal{B}\left(\mathbb{R}^{d}\right)$,

$$
\lim _{|x| \rightarrow \infty} P(t, x, B)=0 .
$$

Proof. (i) This is just [30, Corollary 3.4].

(ii) Assume that $\left(T_{t}\right)_{t \geqslant 0}$ is $C_{b}$-Feller. Then, for any $t>0$ and $f \in C_{\infty}\left(\mathbb{R}^{d}\right), T_{t} f$ is continuous. For any $\varepsilon>0$, we first choose $\delta>0$ such that $|f| \mathbb{1}_{B(0, \delta)^{c}} \leqslant \varepsilon$. Thus, 
for $x \in \mathbb{R}^{d}$,

$$
\begin{aligned}
\left|T_{t} f(x)\right| & \leqslant \int_{B(0, \delta)}|f(y)| P(t, x, d y)+\int_{B(0, \delta)^{c}}|f(y)| P(t, x, d y) \\
& \leqslant\|f\|_{\infty} P(t, x, B(0, \delta))+\varepsilon .
\end{aligned}
$$

Hence,

$$
\lim _{|x| \rightarrow \infty}\left|T_{t} f(x)\right| \leqslant\|f\|_{\infty} \lim _{|x| \rightarrow \infty} P(t, x, B(0, \delta))+\varepsilon=\varepsilon .
$$

Letting $\varepsilon \rightarrow 0$ yields that $T_{t} f \in C_{\infty}\left(\mathbb{R}^{d}\right)$.

On the other hand, for any bounded set $B \in \mathcal{B}\left(\mathbb{R}^{d}\right)$, we can choose some $f \in$ $C_{\infty}\left(\mathbb{R}^{d}\right)$ such that $f \geqslant 0$ and $\left.f\right|_{B} \equiv 1$. Therefore,

$$
T_{t} f(x) \geqslant \int_{B} f(y) P(t, x, d y)=P(t, x, B) .
$$

Since $\left(T_{t}\right)_{t \geqslant 0}$ is $\left(C_{\infty^{-}}\right)$Feller,

$$
0=\lim _{|x| \rightarrow \infty}\left|T_{t} f(x)\right|=\lim _{|x| \rightarrow \infty} T_{t} f(x) \geqslant \lim _{|x| \rightarrow \infty} P(t, x, B) .
$$

We close this section with an abstract result for Feller semigroups.

Proposition 4.6. The martingale problem for $\left(-p(\cdot, D), C_{c}^{\infty}\left(\mathbb{R}^{d}\right)\right)$ is well posed if, and only if, the test functions $C_{c}^{\infty}\left(\mathbb{R}^{d}\right)$ are an operator core for the Feller generator $(A, D(A))$, i.e. $\overline{\left.A\right|_{C_{c}^{\infty}\left(\mathbb{R}^{d}\right)}}=A$.

Proof. Assume that the martingale problem for $\left(-p(\cdot, D), C_{c}^{\infty}\left(\mathbb{R}^{d}\right)\right)$ is well posed. According to a result by van Casteren, [37, Theorem 2.5, Page 283], see also that by Okitaloshima and van Casteren, [25, Theorem 3.1, Page 789], there exists a unique extension $(A, D(A))$ of $\left(-p(\cdot, D), C_{c}^{\infty}\left(\mathbb{R}^{d}\right)\right)$ which is a Feller generator. In particular, $\overline{\left.A\right|_{C_{c}^{\infty}\left(\mathbb{R}^{d}\right)}}=A$.

On the other hand, suppose that the test functions $C_{c}^{\infty}\left(\mathbb{R}^{d}\right)$ are an operator core for the Feller operator $(A, D(A))$. By the Hille-Yosida-Ray Theorem, see e.g. 10, Chapter 4, Theorem 2.2, Page 165], the range $(\lambda+p(\cdot, D))\left(C_{c}^{\infty}\left(\mathbb{R}^{d}\right)\right)$ is dense in $C_{\infty}\left(\mathbb{R}^{d}\right)$ for some $\lambda>0$. Since $\left(-p(\cdot, D), C_{c}^{\infty}\left(\mathbb{R}^{d}\right)\right)$ satisfies the positive maximum principle, it is dissipative in the sense that

$$
\|\lambda u-(-p(\cdot, D)) u\|_{\infty} \geqslant \lambda\|u\|_{\infty} \quad \text { for all } u \in C_{c}^{\infty}\left(\mathbb{R}^{d}\right)
$$

cf. [10, Chapter 4, Theorem 2.1, Page 165]. Therefore, the well-posedness of the martingale problem for $\left(-p(\cdot, D), C_{c}^{\infty}\left(\mathbb{R}^{d}\right)\right)$ follows from [10, Chapter 4 , Theorem 4.1, Page 182].

\section{ACKNOWLEDGEMENTS}

Financial support through DFG (grant Schi 419/5-2) and DAAD (PPP Kroatien) (for the first author), the Alexander-von-Humboldt Foundation and the Programme of Excellent Young Talents in Universities of Fujian (Nos. JA10058 and JA11051) (for the second author) is gratefully acknowledged. Most of this work was done when the second author was a Humboldt fellow at TU Dresden. He is grateful for the hospitality and the good working conditions. We are grateful to an anonymous referee whose comments helped to improve the exposition of this paper. 


\section{REFERENCES}

[1] Bass, R.F.: Uniqueness in law for pure jump type Markov processes, Probab. Theory Related Fields, 79 (1988), 271-287. MR.958291 (89h:60118)

[2] Bass, R.F.: Occupation time densities for stable-like processes and other pure jump Markov processes, Stoch. Proc. Appl., 29 (1988), 65-83. MR.952820 (89i:60142)

[3] Berg, C. and Forst, G.: Potential Theory on Locally Compact Abelian Groups, SpringerVerlag, Berlin, 1975. MR0481057 (58:1204)

[4] Berman, S.M.: Local times and sample function properties of stationary Gaussian processes, Trans. Amer. Math. Soc., 137 (1969), 277-299. MR0239652 (39:1009)

[5] Bertoin, J.: Lévy Processes, Cambridge Univ. Press, Cambridge 1996. MR.1406564 (98e:60117)

[6] Böttcher, B.: An overshoot approach to recurrence and transience of Markov processes, Stoch. Proc. Appl., 121 (2011), 1962-1981.

[7] Chung, K.L. and Fuchs, W.H.J.: On the distribution of values of sums of random variables, Memoirs Amer. Math. Soc. 6 (1951), 1-12. MR0040610 (12:722e)

[8] Courrège, Ph.: Sur la forme intégro-différentielle des opérateurs de $C_{K}^{\infty}$ dans $C$ satisfaisant au principe du maximum, Sém. Théorie du Potentiel (1965/66) exposé 2, 38 pp.

[9] Dynkin, E.B.: Markov Processes (2 vols.), Springer, Berlin, 1965.

[10] Ethier, S.E. and Kurtz, T.G.: Markov Processes: Characterization and Convergence, Wiley, Series in Probab. and Math. Stat., New York, 1986. MR838085 (88a:60130)

[11] Evans, K.P. and Jacob, N.: Feller semigroups obtained by varibale order subordination, Rev. Math. Complut. 20 (2007), 293-307. MR2351111(2009i:35361)

[12] Fristedt, B.: Sample functions of stochastic processes with stationary, independent increments, in: Ney, P. and S. Port: Advances in Probability and Related Topics vol. 3, Marcel Dekker, New York, 1974, 241-396. MR0400406 (53:4240)

[13] Getoor, R.K. and Kesten, H.: Continuity of local times for Markov processes, Compositio Math. 24 (1972), 277-303. MR0310977(46:10075)

[14] Hartman, P. and Wintner, A.: On the infinitesimal generators of integral convolutions, Amer. J. Math. 64 (1942), 273-298. MR0006635 (4:18a)

[15] Hawkes, J.: Potential theory of Lévy processes. Proc. London Math. Soc. 38 (1979), 335-352. MR531166 (80g:60077)

[16] Hawkes, J.: Local times as stationary processes, in: K.D. Ellworthy (ed.): From local times to global geometry, control and physics. Pitman Research Notes in Math., vol. 150, Longman, Chicago, 1986, 111-120. MR894527 (88g:60189)

[17] Jacob, N.: Characteristic functions and symbols in the theory of Feller processes, Potential Analysis 8 (1998), 61-68. MR.1608650 (99b:60121)

[18] Jacob, N.: Pseudodifferential Operators and Markov Processes (3 vols), Imperial College Press, London, 2001, 2002, 2005. MR.1873235 (2003a:47104) MR.1917230||(2003k:47077). MR2158336 (2006i:60001)

[19] Jacob, N. and Schilling, R.L.: Estimates for Feller semigroups generated by pseudodifferential operators, in: J. Rákosník (ed.): Function Spaces, Differential Operators and Nonlinear Analysis. Proceedings of the Conference, Paseky nad Jizerou, September 3-9, 1995, Prometheus Publishing House 1996, 27-49. MR1480928 (98k:47084)

[20] Jacob, N. and Schilling, R.L.: Lévy-type processes and pseudodifferential operators, in: Barndorff-Nielsen, O.E. et al. (eds.): Lévy Processes: Theory and Applications, Birkhäuser, Boston, 2001, 139-168. MR.1833696 (2002c:60077)

[21] Knopova, V. and Schilling, R.L.: A note on the existence of transition probability densities for Lévy processes, to appear in Forum Math., 2011. See also arXiv 1003.1419v2.

[22] Kolokoltsov, V.: Symmetric stable laws and stable-like jump-diffusion, Proc. London Math. Soc., 80 (2000), 725-768. MR:1744782 (2001f:60016)

[23] Meyn, S.P. and Tweedie, R.: Generalized resolvents and Harris recurrence of Markov processes, Contemp. Math., 149 (1993), 227-250. MR1229967 (94i:60075)

[24] Meyn, S.P. and Tweedie, R.: Stability of Markovian processes II: Continuous-time processes and sampled chains, Adv. Appl. Prob., 24 (1993), 487-517. MR:1234294 (94g:60136)

[25] Okitaloshima, O. and van Casteren, J.A.: On the uniqueness of the martingale problem, Int. J. Math., 7 (1996), 775-810. MR1417786 (97i:60096) 
[26] Port, S.C. and Stone, C.J.: Infinitely divisible processes and their potential theory I, II, Ann. Inst. Fourier 21 (2) (1971), 157-275, and Ann. Inst. Fourier 21 (4) (1971), 179-265. MR0346919(49:11640)

[27] Sato, K.: Lévy processes and Infinitely Divisible Distributions, Cambridge University Press, Studies Adv. Math., 68, Cambridge, 1999. MR.1739520 (2003b:60064)

[28] Schilling, R.L.: Feller processes generated by pseudo differential operators: On the Hausdorff dimension of their sample paths, J. Theor. Probab. 11 (1998), 303-330. MR 1622573 (99c:60082)

[29] Schilling, R.L.: Conservativeness of semigroups generated by pseudodifferential operators, Potential Analysis 9 (1998), 91-104. MR.1644108 (99g:60132)

[30] Schilling, R.L.: Conservativeness and extensions of Feller semigroups, Positivity 2 (1998), 239-256. MR 1653474 (2000c:47085)

[31] Schilling, R.L.: Growth and Hölder conditions for the sample paths of Feller processes, Probab. Theor. Related Fields 112 (1998), 565-611. MR1664705 (99m:60131)

[32] Schilling, R.L. and Schnurr, A.: The symbol associated with the solution of a stochastic differential equation, Elect. J. Probab., 15 (2010), 1369-1393. MR2721050

[33] Schilling, R.L., Song, R.M. and Vondraček, Z.: Bernstein Functions. Theory and Applications, de Gruyter, Berlin 2010. MR2598208 (2011d:60060)

[34] Schilling, R.L. and Wang, J.: Strong Feller continuity of Feller processes and semigroups, Preprint, to appear in Infinit. Dimens. Anal. Quant. Probab. Related Topics, 2012. See also arXiv:1006.5286

[35] Taylor, M.E.: Pseudodifferential Operators, Princeton University Press, Princeton, NJ, 1981. MR618463(82i:35172)

[36] Tweedie, R.: Topological conditions enabling use of Harris methods in discrete and continuous time, Acta Appl. Math., 34 (1994), 175-188. MR1273853(95d:60112)

[37] van Casteren, J.A.: On martingales and Feller semigroups, Results in Math., 21 (1992), 274-288. MR.1157331 (93h:60115)

[38] Wang, F.-Y.: Functional Inequalities, Markov Processes and Spectral Theory, Science Press, Beijing, 2005.

[39] Wang, J.: Stability of Markov processes generated by Lévy type operators, Chinese Ann. Math. Ser. A. (in Chinese) 32 (2011), 33-50; Chinese J. Contemp. Math. (in English), 32 (2011), 33-52. MR2663819

Institut für Mathematische Stochastik, TU Dresden, 01062 Dresden, Germany

E-mail address: rene.schilling@tu-dresden.de

School of Mathematics and Computer Science, Fujian Normal University, 350007, Fuzhou, People's Republic of China

E-mail address: jianwang@fjnu.edu.cn 